\title{
Further implications of a computational model of septohippocampal cholinergic modulation in eyeblink conditioning
}

\author{
CATHERINE E. MYERS and BRANDON R. ERMITA \\ Rutgers University, Newark, New Jersey \\ MICHAEL HASSELMO \\ Harvard University, Cambridge, Massachusetts \\ and \\ MARK A. GLUCK \\ Rutgers University, Newark, New Jersey
}

\begin{abstract}
Previously we have shown that Gluck and Myers's (1993) corticohippocampal model could be extended to incorporate Hasselmo and Schnell's (1994) hypothesis that septohippocampal cholinergic processes regulate the amount of information storage in hippocampus. The generalized model could account for the effect of the anticholinergic drug scopolamine in delaying onset of eyeblink conditioning (Myers et al., 1996). Here, we show that the model also accounts for additional eyeblink results, including quick recovery after scopolamine is removed, preserved latent inhibition, learned irrelevance and extinction under scopolamine, and no effect of systemic scopolamine after hippocampal lesion. Additionally, the model is consistent with data concerning localized scopolamine injections to the medial septum, the lateral septum, and the hippocampus and their effect on eyeblink conditioning.
\end{abstract}

In a previous paper, we showed that an existing model of corticohippocampal interaction in associative learning could be generalized to include the effects of cholinergic disruption (Myers et al., 1996). The corticohippocampal model assumes that the hippocampal regionincluding the hippocampus, the dentate gyrus, the subiculum, and the entorhinal cortex - is necessary for adaptively modifying stimulus representations so as to compress redundant information but differentiate predictive information. By contrast, long-term storage areas in the cerebellum are assumed to be limited to simpler forms of learning that do not include these representational modifications (Gluck \& Myers, 1993). This model accounts for a range of data on associative learning in intact and hippocampal-lesioned animals (Gluck \& Myers, 1993; Myers \& Gluck, 1994, 1996; Myers, Gluck, \& Granger, 1995).

Associative learning is also affected by disruption of the cholinergic pathways from the medial septum to the hippocampus. Hasselmo and Schnell (1994) hypothesized that septohippocampal cholinergic input modulates hippocampal dynamics between information storage and infor-

This work was funded by the Office of Naval Research through the Young Investigator Program (M.A.G.) and Grant N00014-88-K-0112 (M.A.G.) and by a grant from the McDonnel-Pew Foundation Program in Cognitive Neuroscience (M.A.G. and C.E.M.). Correspondence concerning this article should be addressed to C. E. Myers, Center for Molecular and Behavioral Neuroscience, Rutgers University, Newark, NJ 07102 (e-mail: myers@pavlov. rutgers.edu). mation recall. This hypothesis is consistent with behavioral and physiological data (Hasselmo \& Schnell, 1994) and can be implemented in the corticohippocampal model by assuming that the hippocampal region network's learning rate is determined by the degree of septohippocampal cholinergic input (Myers et al., 1996). This assumption does not add any additional complexity to the model and suffices to account for data showing that the acquisition of a classically conditioned response is slowed but not abolished after cholinergic disruption in animals (Solomon, Solomon, van der Schaaf, \& Perry, 1983) and in humans (Solomon et al., 1993). The model also correctly accounts for the facilitated learning that follows the administration of cholinergic agonists - as well as the fact that this facilitation is dose-dependent and that higher doses may actually retard learning (Myers et al., 1996).

However, there are many other possible manipulations to the corticohippocampal model that could result in slowed acquisition of conditioned responding and so mimic the effects of cholinergic disruption in the modelalthough they do not correspond as readily to a physiological mechanism, such as that proposed by Hasselmo and Schnell (1994). If the assumption that septohippocampal cholinergic modulation determines hippocampal region learning rates is valid, it should also account for other effects of cholinergic disruption on eyeblink conditioning. Accordingly, this paper considers a wider range of data regarding the effects of cholinergic drugs on associative learning and shows that the model can account for these effects as well; this, in turn, strengthens the argu- 
ment that the model provides a useful description of these aspects of septohippocampal cholinergic modulation.

\section{BACKGROUND: \\ THE CORTICOHIPPOCAMPAL MODEL}

Classical eyeblink conditioning is a canonical preparation for studying associative learning in a variety of species including rabbits (see, e.g., Gormezano, Kehoe, \& Marshall, 1983) and humans (see, e.g., Solomon et al., 1993; Woodruff-Pak, 1993). A previously neutral tone or light (the conditioned stimulus, or CS) is repeatedly paired with a blink-evoking corneal airpuff or paraorbital shock (the unconditioned stimulus, or US) until presentation of the CS alone evokes a protective eyeblink (the conditioned response, or CR). The anatomical substrates for this learning system are well understood. Thompson (1986) has proposed that CS information from the pontine nuclei travels via mossy fibers to the cerebellar Purkinje cells and interpositus nuclei, which, in turn, produce output driving the behavioral CR. Plasticity is assumed to occur at the sites where CS information converges on the Purkinje cells and the interpositus nuclei. Thompson (1986) further proposed that this learning is driven by an error signal, computed by the inferior olive, which reflects the difference between the US and CR. This signal then travels to the Purkinje cells and interpositus nuclei and modifies synapse strength according to conjoint presynaptic and postsynaptic activity and in proportion to the error. This learning rule is formalized as the least mean square (LMS) algorithm (Widrow \& Hoff, 1960) and has relations both to biological plasticity mechanisms such as long-term potentiation and depression (see, e.g., Levy, Brassel, \& Moore, 1983) and to mathematical specifications of conditioning (Rescorla \& Wagner, 1972).

The Thompson (1986) model of the cerebellar substrates of eyeblink conditioning can be easily formalized in a connectionist network such as the one shown on the right in Figure 1A (Gluck, Myers, \& Thompson, 1994). This network learns to map from inputs specifying the presence of CSs, as well as contextual or background stimuli, to a pattern of activations in an internal or hidden layer of nodes. These activation patterns, which we term a re-representation of the input patterns, are then mapped to output representing the behavioral CR. The LMS rule can be used to modify the weights between the internal layer and output nodes. It cannot, however, be used to modify the weights between the inputs and the internal layer nodes, since the error is not defined for the internal layer nodes. That is to say, the representation is fixed.

Gluck and Myers (1993) suggested a way in which hippocampal region processing could be used to modify stim-
(A) Cortical
Network

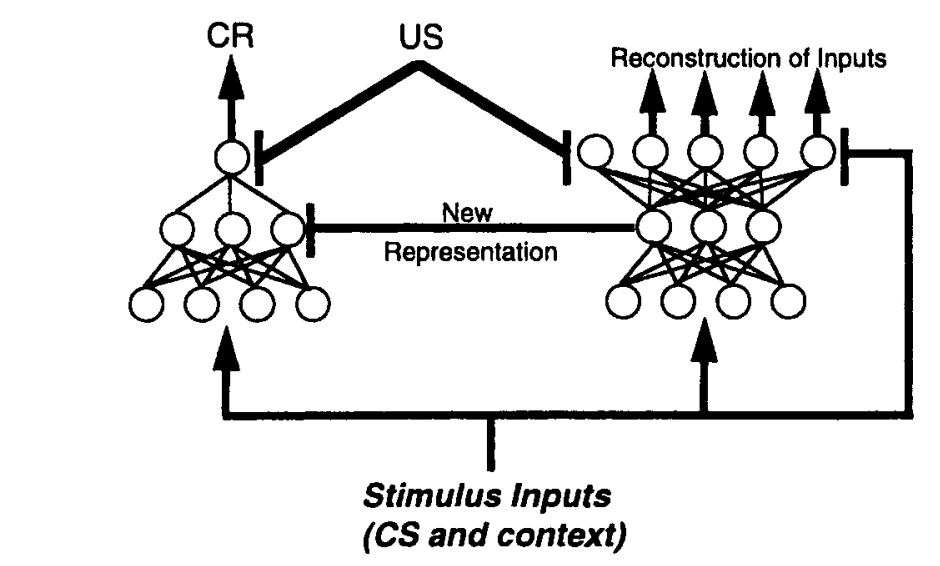

Hippocampal-
Region

Region
Network
(B)
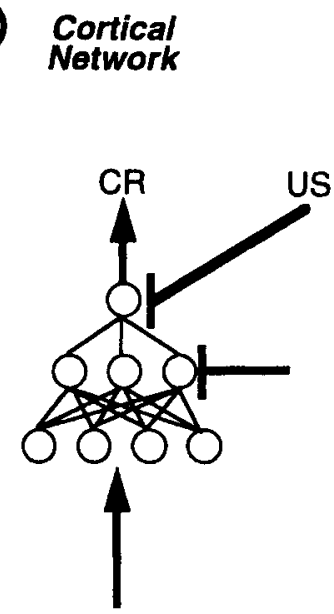

Stimulus Inputs

(CS and context)

Figure 1. The corticohippocampal model (Gluck \& Myers, 1993). (A) The intact model. One network representing hippocampal region processing, on the right, learns to map from stimulus inputs through a narrow internal layer to outputs that reconstruct that input and also predict unconditioned stimulus arrival. In the process it forms new representations in its internal layer that compress redundancies while preserving predictive information. A network representing long-term storage sites in the cerebellum, on the left, cannot form new representations in this way but can adopt the representations formed in the hippocampal region network and then learn a mapping from those representations to a conditioned response output (CR). (B) The lesioned model. Hippocampal region damage is simulated by disabling the hippocampal region network and fixing the lower layer of weights in the cerebellar network. The cerebellar network can still learn new behavioral responses by mapping from its preexisting (and now fixed) internal layer representations to a CR. From "A Computational Model of Cholinergic Disruption of Septohippocampal Activity in Classical Eyeblink Conditioning," by C. E. Myers, B. R. Ermita, K. Harris, M. Hasselmo, P. Solomon, and M. A. Gluck, 1996, Neurobiology of Learning \& Memory, 66, pp. 51-66. Copyright 1996 by the American Psychological Association, Inc. Reprinted with permission. 
ulus representations to facilitate learning. In particular, if two CSs are redundant or should be mapped to similar $\mathrm{CRs}$, their representations should be compressed or made more similar. Conversely, two CSs that are to be mapped to different CRs should have differentiated, or highly dissimilar, representations. Gluck and Myers suggested that the hippocampal region can do this representational modification and can provide this information to modulate learning in the cerebellum and elsewhere.

This basic theory has been implemented in a connectionist model, as shown in Figure 1, and applied to eyeblink conditioning (Gluck \& Myers, 1993). Hippocampal region processing is implemented through a predictive autoencoder (Baldi \& Hornik, 1989; Hinton, 1989), which learns to transform CS inputs, through a narrow internal node layer, into outputs that reconstruct those inputs and also predict the US. Because the internal layer in this network contains fewer nodes than do the input and output layers, the network is forced to compress redundant information while preserving and differentiating information that predicts the US. A training algorithm that accomplishes this is error backpropagation (Rumelhart, Hinton, \& Williams, 1986), although other algorithms may be more biologically plausible (see Myers et al., 1995, but also Schmajuk \& DiCarlo, 1992). The result is that, over time, representations develop in the internal layer that have exactly the properties required by the hypothesis.

This hippocampal region network then provides these new representations to the cerebellar network. A random recoding of the hippocampal region network's internal layer activations becomes the desired output for each cerebellar network internal layer node, and the error is the difference between this and the node's actual output. The cerebellar network can then use the LMS rule to adapt its lower layer weights, just as it uses the LMS rule to adapt its upper layer weights. Over time, representations develop in the cerebellar network internal layer nodes that are linear recombinations of those developed by the hippocampal region network. The cerebellar network learns to map from these acquired representations to the desired behavioral responses.

This two-module intact system (Figure 1A) is assumed to model eyeblink conditioning in the intact (normal) animal. Broad hippocampal region damage is simulated by disabling the hippocampal region network (Figure 1B). In this lesioned model, no new hippocampal-dependent representations are formed, and the training signal to the cerebellar network is silenced. The cerebellar network can adopt no new representations, although it can still learn to map from its existing representations to new behavioral responses.

Hippocampal region damage does not impair conditioned eyeblink responding in humans (Daum, Channon, \& Canavan, 1989; Gabrieli et al., 1995; Weiskrantz \& Warrington, 1979; Woodruff-Pak, 1993), rabbits (Akase, Alkon, \& Disterhoft, 1989; Solomon, 1977; Solomon \& Moore, 1975) or rats (Schmajuk, Lam, \& Christiansen, 1994). Figures $2 A$ and $2 B$ summarize these data. Figure $2 \mathrm{C}$ shows learning of a simple $\mathrm{CS}-\mathrm{US}$ association in the intact and lesioned models. In the lesioned model, there is no new formation of stimulus representations, but, for a simple CS-US association, any preexisting rep-
(A)

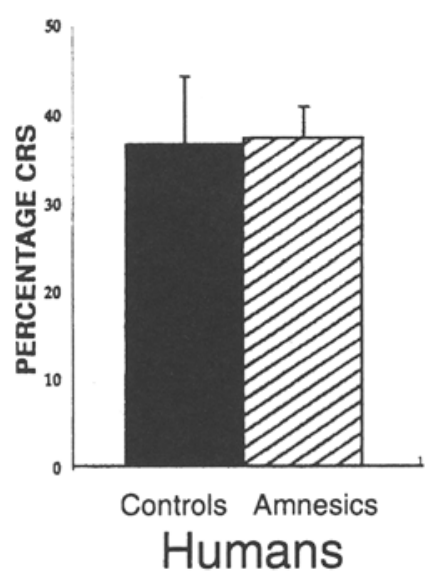

(B)

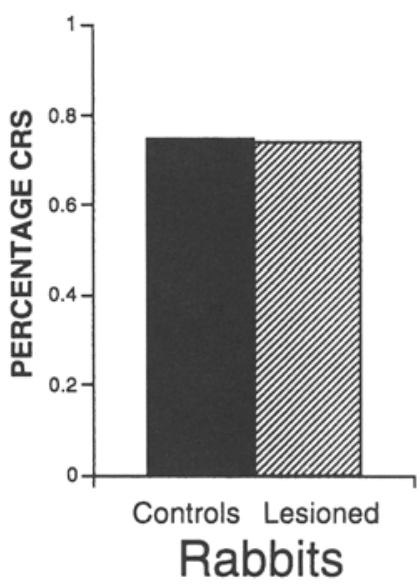

(C)

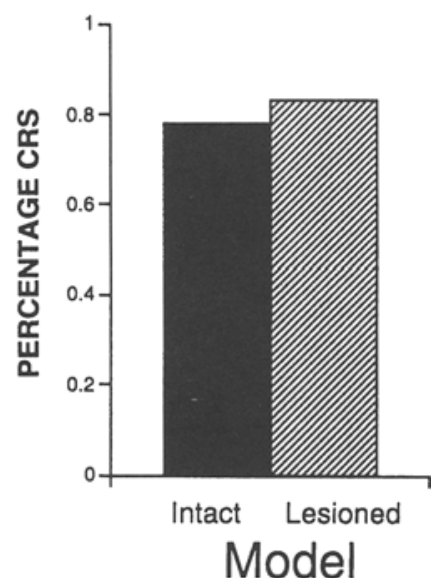

Figure 2. Hippocampal region damage does not impair acquisition of conditioned eyeblink responding in (A) humans (Gabrieli et al., 1995) or (B) rabbits (Solomon \& Moore, 1975), measured as percent of trials generating conditioned responses after equal amounts of training. $(C)$ The lesioned model also learns conditioned stimulusunconditioned stimulus associations as quickly as the intact model. From "A Computational Model of Cholinergic Disruption of Septohippocampal Activity in Classical Eyeblink Conditioning," by C. E. Myers, B. R. Ermita, K. Harris, M. Hasselmo, P. Solomon, and M. A. Gluck, 1996, Neurobiology of Learning \& Memory, 66, pp. $51-66$. Copyright 1996 by the American Psychological Association, Inc. Reprinted with permission. 
resentation is likely to suffice. Thus, the lesioned model correctly shows no deficit in CS-US learning (Gluck \& Myers, 1993).

By contrast with the situation for simple acquisition, Gluck and Myers's (1993) theory predicts that the hippocampal region will be critical if new stimulus representations are required. This is expected to occur in paradigms such as sensory preconditioning, in which prior unreinforced exposure to a two-cue compound increases the rate at which subsequent learning to one of the cues generalizes to the other cue (Thompson, 1972). In the intact model, a similar effect obtains. When the two cues are presented together in the first phase, their representations are compressed, which increases generalization between them. Thus, when one of those cues is subsequently paired with a US, this learning tends to transfer to the second cue as well (Gluck \& Myers, 1993). In the lesioned model, with no such representational compression, there is no effect of sensory preconditioning. This is consistent with data showing that hippocampal region damage eliminates sensory preconditioning in rabbit eyeblink conditioning (Port \& Patterson, 1984). Similarly, the model accounts for many other trial level conditioning effects in intact and hippocampal-lesioned animals (Gluck \& Myers, 1993; Myers \& Gluck, 1994; Myers et al., 1995) and has even been applied to other domains, such as human category learning (Gluck, Oliver, \& Myers, 1996) and rodent odor discrimination (Myers \& Gluck, 1996).

\section{SEPTOHIPPOCAMPAL CHOLINERGIC MODULATION IN THE CORTICOHIPPOCAMPAL MODEL}

The corticohippocampal model has been extended to include a role for septohippocampal cholinergic inputs (Myers et al., 1996). These cholinergic inputs enter the hippocampus through the fornix, along with other presumably modulatory neurotransmitters (Mosko, Lynch, $\&$ Cotman, 1973). Acetylcholine from the basal forebrain has several neuromodulatory effects on glutamatergic and GABAergic neurotransmission in the hippocampus and cortex, including suppression of synaptic transmission (Hasselmo \& Schnell, 1994), enhancement of pyramidal cell excitability through suppression of currents underlying adaptation (Barkai \& Hasselmo, 1994; Madison, Lancaster, \& Nicoll, 1987), and enhancement of synaptic modification (Hasselmo \& Barkai, 1995; Huerta \& Lisman, 1993).

Cholinergic suppression of synaptic transmission in the hippocampus and elsewhere is laminarly selective, affecting some kinds of synapses more strongly than others. For example, acetylcholine strongly blocks intrinsic recurrent collaterals in the stratum radiatum of hippocampal field CA.3 but has a much weaker effect on external inputs (Hasselmo, Schnell, \& Barkai, 1995). Hasselmo (1995) has interpreted these data as suggesting a means whereby acetylcholine could modulate hippocampal dynamics along a continuum between modes for storing new information versus recalling previously stored information. In particular, when a stimulus input is presented to the hippocampus, a subpopulation of nodes will be activated; this activation feeds through the intrinsic collaterals and activates other nodes that have previously been associated. For example, if nodes $A$ and $B$ have previously been coactive, so that an association forms between them, later presentation of A alone may cause activity in the collaterals that activates $\mathrm{B}$ as well. However, when a new pattern is to be stored (say, A and C), this collateral activity must be suppressed. Hasselmo and Schnell (1994) have proposed that the presence of acetylcholine suppresses these recurrent collaterals, while enhancing plasticity, to encourage storage. Thus, cholinergic inputs can modulate the hippocampus along a continuum between storage and recall states. Consistent with this account, microdialysis techniques-used to measure and quantify local changes in neurotransmitter levels-verify that acetylcholine in the ventral hippocampus increases while a CS-US association is being learned and decreases after the CR is well learned (Meyer, Allen, \& Yokel, 1996).

According to Hasselmo's (1995) account, disrupting septohippocampal acetylcholine through septal lesion or reducing it through anticholinergic drugs should greatly disrupt hippocampal function by reducing the ability to store information and increasing the tendency to recall old memories when presented with new ones. In fact, septal lesion does disrupt hippocampal processing behaviorally and greatly retards acquisition of conditioned eyeblinking in rabbits (Berry \& Thompson, 1979; Powell, Milligan, \& Buchanan, 1976; Salvatierra \& Berry, 1989), as is shown in Figure 3A. Septal lesion, of course, interrupts all septohippocampal connections, including GABAergic projections that may themselves play an important role in modulating hippocampal activity (Berry \& Thompson, 1979; Buzsáki \& Eidelberg, 1983). We will return to discuss the possible role of septohippocampal GABAergic inputs later.

Systemic administration of scopolamine, a drug that blocks muscarinic cholinergic receptors (Brazhnik, Vinogradova, Stafekhina, \& Kitchigina, 1993), also greatly retards eyeblink conditioning in humans (Bahro, Schreurs, Sunderland, \& Molchan, 1995; Solomon et al., 1993) and rabbits (Solomon et al., 1983), as is shown in Figures 3B and 3C. Systemic scopolamine also interrupts cholinergic processes throughout the brain, such as those arising from the nucleus basalis and the pons and midbrain tegmentum and those targeting cholinergic interneurons of the striatum and cortex (Izquierdo, 1989); however, these other afferents and targets appear to be of lesser importance in eyeblink conditioning (Ginn \& Powell, 1992; see also Kesner, 1988), suggesting that the septohippocampal pathways are the critical ones.

Importantly, neither medial septal lesion nor scopolamine significantly decreases the magnitude of the unconditioned response (see, e.g., Lockhart \& Moore, 1975; Moore, Goodell, \& Solomon, 1976). If the effect of these 
(A)

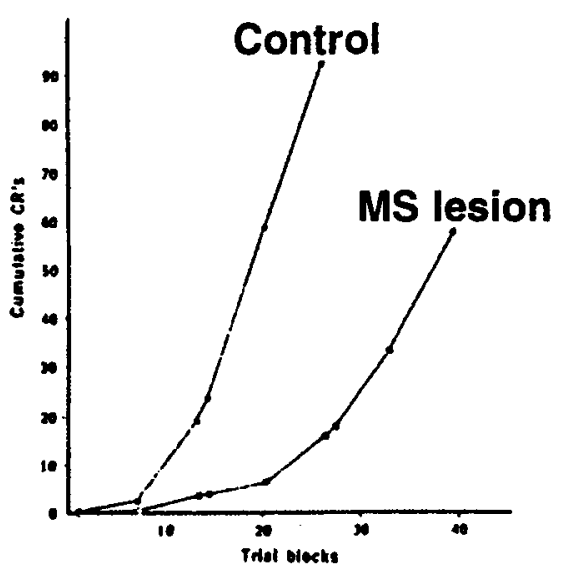

(B)

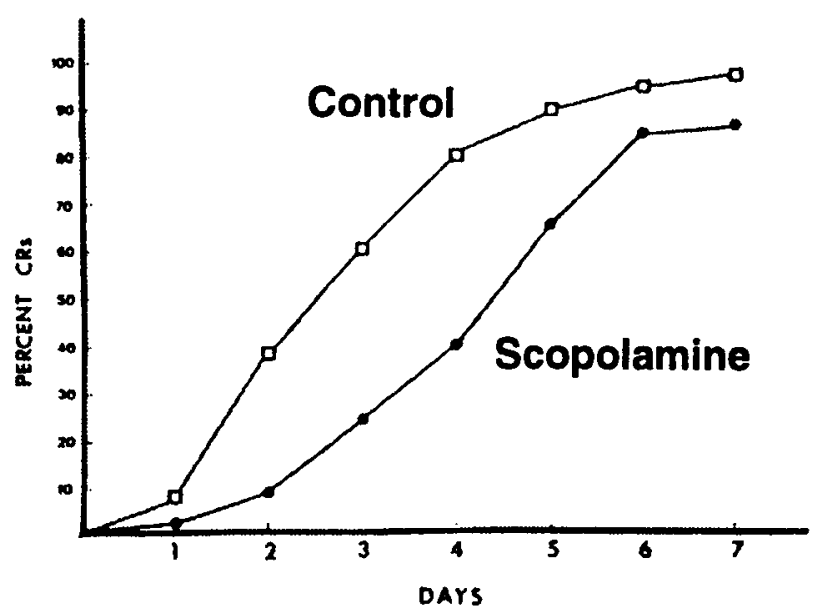

(C)

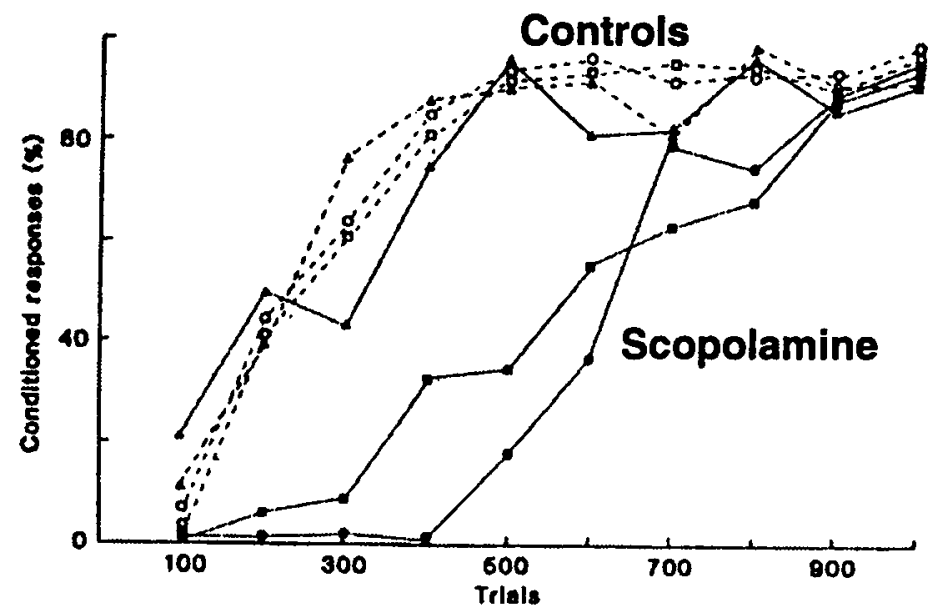

Figure 3. Acquisition of conditioned eyeblink responding is impaired by (A) medial septal lesion in rabbits (Solomon \& Gottfried, 1981) and also by systemic administration of the anticholinergic scopolamine in (B) rabbits (Solomon et al., 1983) and (C) humans (Solomon et al., 1993). From "A Computational Model of Cholinergic Disruption of Septohippocampal Activity in Classical Eyeblink Conditioning," by C. E. Myers, B. R. Ermita, K. Harris, M. Hasselmo, P. Solomon, and M. A. Gluck, 1996, Neurobiology of Learning \& Memory, 66, pp. 51-66. Copyright 1996 by the American Psychological Association, Inc. Reprinted with permission.

manipulations was to reduce unconditioned responding, that should cause a generalized motor deficit- -with animals failing to give $\mathrm{CRs}$, not because they were unable to learn the CS-US association, but merely because they were unable to express that learning through a blink response. Far from causing such a motor deficit, scopolamine generally causes motor hyperactivity (Campbell, Lytle, \& Fibiger, 1969). Similarly, CS sensitivity is not decreased to a point that ought to interfere with conditioning (see, e.g., Moore et al., 1976). Thus, the slow learning under scopolamine appears specifically to reflect an associative learning deficit (see Spencer \& Lal, 1983)--presumably by disrupting septohippocampal cholinergic processes.
Importantly, the effect of medial septal lesion and cholinergic disruption is not to completely abolish eyeblink conditioning but merely to delay its onset. Once conditioning begins to emerge, learning proceeds at a nearnormal rate and eventually reaches normal asymptotic levels (Figure 3 ). All of these data contrast with the effects of outright hippocampal region damage, which does not affect the acquisition of simple eyeblink conditioning (refer to Figures $2 \mathrm{~A}$ and $\mathrm{B}$ ).

Hasselmo and Schnell's (1994) idea that septohippocampal cholinergic processes regulate the amount of hippocampal storage can be implemented in the corticohippocampal model by assuming that the learning rate of the hippocampal region network is modulated by septo- 
(A) Cortical Network $\begin{gathered}\text { Hippocampal- } \\ \text { Region } \\ \text { Network }\end{gathered}$

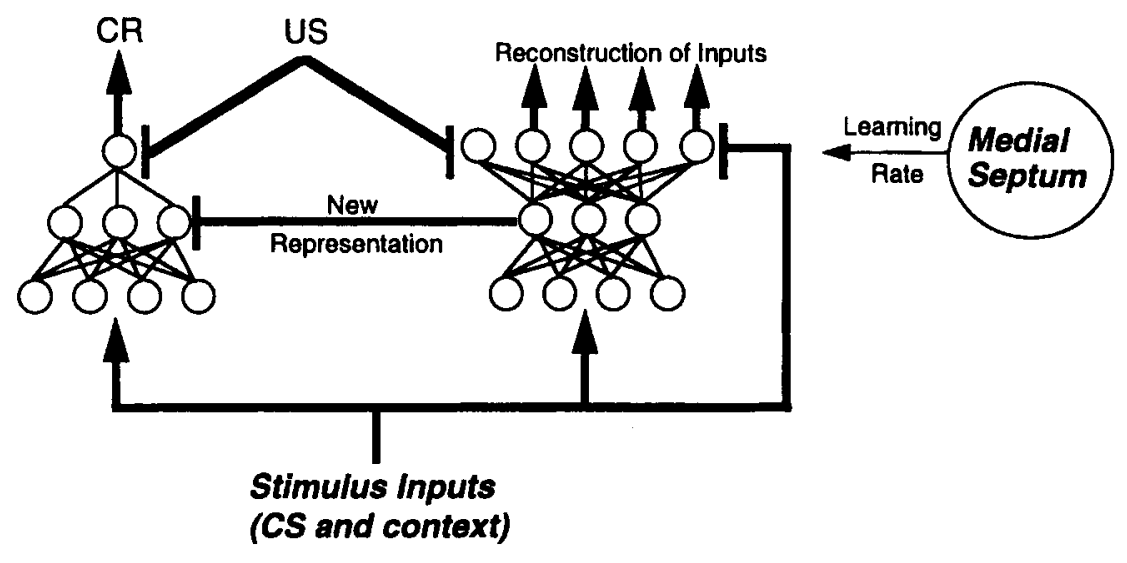

(B)

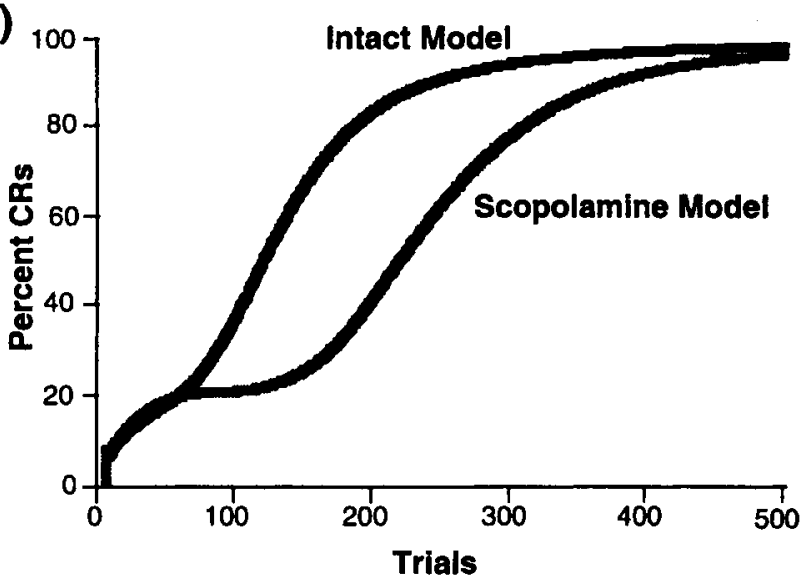

Figure 4. (A) Hasselmo and Schnell's (1994) hypothesis that septohippocampal cholinergic projections modulate the amount of hippocampal storage can be implemented in the corticohippocampal model by assuming that the medial septum determines the hippocampal region network's learning rate (Myers et al., 1996). (B) Conditioned stimulus-unconditioned stimulus learning in the intact and scopolamine models. The effect of scopolamine in the model is to delay the onset of conditioned responding, much like the effects of the anticholinergic scopolamine in vivo. From " $\mathrm{A} \mathrm{Com-}$ putational Model of Cholinergic Disruption of Septohippocampal Activity in Classical Eyeblink Conditioning," by C. E. Myers, B. R. Ermita, K. Harris, M. Hasselmo, P. Solomon, and M. A. Gluck, 1996, Neurobiology of Learning \& Memory, 66, pp. 51-66. Copyright 1996 by the American Psychological Association, Inc. Reprinted with permission.

hippocampal cholinergic input. This learning rate is independent of the learning rate in the cerebellar network and also independent of the rate at which information is transferred between the networks. Adjusting the hippocampal region learning rate is formally equivalent to manipulating the amount of hippocampal region storage but not of hippocampal region recall or cerebellar storage (see Myers et al., 1996, for formal proof). The resulting scopolamine model is shown in Figure 4A; it is equivalent to the intact model of Figure 1A, except for a reduced learning rate in the hippocampal region network. (Implementation details of the intact, lesioned, and scopolamine models are given in the appendix.)
The scopolamine model can still learn a CS-US association, but it learns more slowly, as shown in Figure 4B. In the scopolamine model, the learning curve is qualitatively the same as that in the intact model, but it is shifted right, representing a delayed onset of behavioral responding. This is followed by a period of learning at approximately normal speed and to normal asymptote. A similar effect is seen in rabbit eyeblink conditioning under scopolamine (Figures 3B and $3 \mathrm{C}$ ). The scopolamine model shows delayed onset of learning, because the cerebellar network can only begin to learn the correct response once it has acquired a stable set of representations from the hippocampal region network; if the hippocampal region 
network's learning is slowed, this will take longer to begin. Once the hippocampal region network's representations are stable and transferred to the cortex, the cortex can begin to learn the behavioral response at the normal rate.

This delayed onset of learning followed by normal learning rates is consistent with Prokasy's (1972) twostage model of classical conditioning. Prokasy proposed that a period during which the behavioral response was adapted toward its asymptote was preceded by a stage in which the response remained relatively constant at its baseline level. Both the baseline level and the duration of this first stage were assumed to vary with individuals. The corticohippocampal model suggests that the effect of scopolamine is to extend the first stage of conditioning, without affecting the second stage, which results in the right-shifted learning curves in the scopolamine model (Figure 4B) and in animals given scopolamine (Figures 3B and 3C). Thompson et al. (1980) proposed that medial septal lesion slowed learning in exactly the same way, by extending the first stage of conditioning.

Just as lowering the hippocampal region learning rate slows learning, raising the learning rate slightly can improve learning; the performance of such a model of cholinergic agonists is shown in Figure 5A. The model expects that cholinergic agonists should similarly speed eyeblink conditioning in animals. In fact, this seems to be the case: Eyeblink conditioning is enhanced in aged rabbits by the cholinesterase inhibitor metrifonate (KronforstCollins et al., 1997).

Figure 5A also shows that raising the hippocampal region network's learning rate beyond some optimal level actually retards learning in the model. This is a general property of connectionist networks (see Jacobs, 1988), resulting when learning fluctuates too greatly to stabilize. This suggests that, beyond some optimal dosage, cholinergic agonists should likewise result in impaired learning.

The prediction of a $U$-shaped dose response curve after treatment with cholinergic agonists (Myers et al., 1996) has recently been confirmed in rabbit eyeblink conditioning (Kronforst-Collins et al., 1997). Kronforst-Collins et al. found that a moderate dose of $12-\mathrm{mg} / \mathrm{kg}$ metrifonate accelerated eyeblink conditioning, whereas doses of $6 \mathrm{mg} / \mathrm{kg}$ and $24 \mathrm{mg} / \mathrm{kg}$ did not (Figure 5B). In other preparations, it has occasionally been shown that moderate doses of cholinergic agonists such as physostigmine can improve learning, whereas very large doses can actually produce impairments (Dumery, Derer, \& Blozovski, 1988; Ennaceur \& Meliani, 1992; Miyamoto, Narumi, Nagaoka, \& Coyle, 1989). The model therefore provides a possible account of this U-shaped dose response curve, which was previously a problematic clinical phenomenon.

\section{MODELING A WIDER RANGE OF SCOPOLAMINE EFFECTS}

The fact that the model accounts correctly for the data regarding effects of cholinergic antagonists and agonists on conditioned acquisition suggests that it is a promising approach. A further strength is the fact that its postulated septohippocampal cholinergic function is predicated on a prior hypothesis that incorporates both behavioral and physiological data. However, there are other parameters in the model that could be adjusted to modulate learning speed. These include cerebellar learning rates, rates of
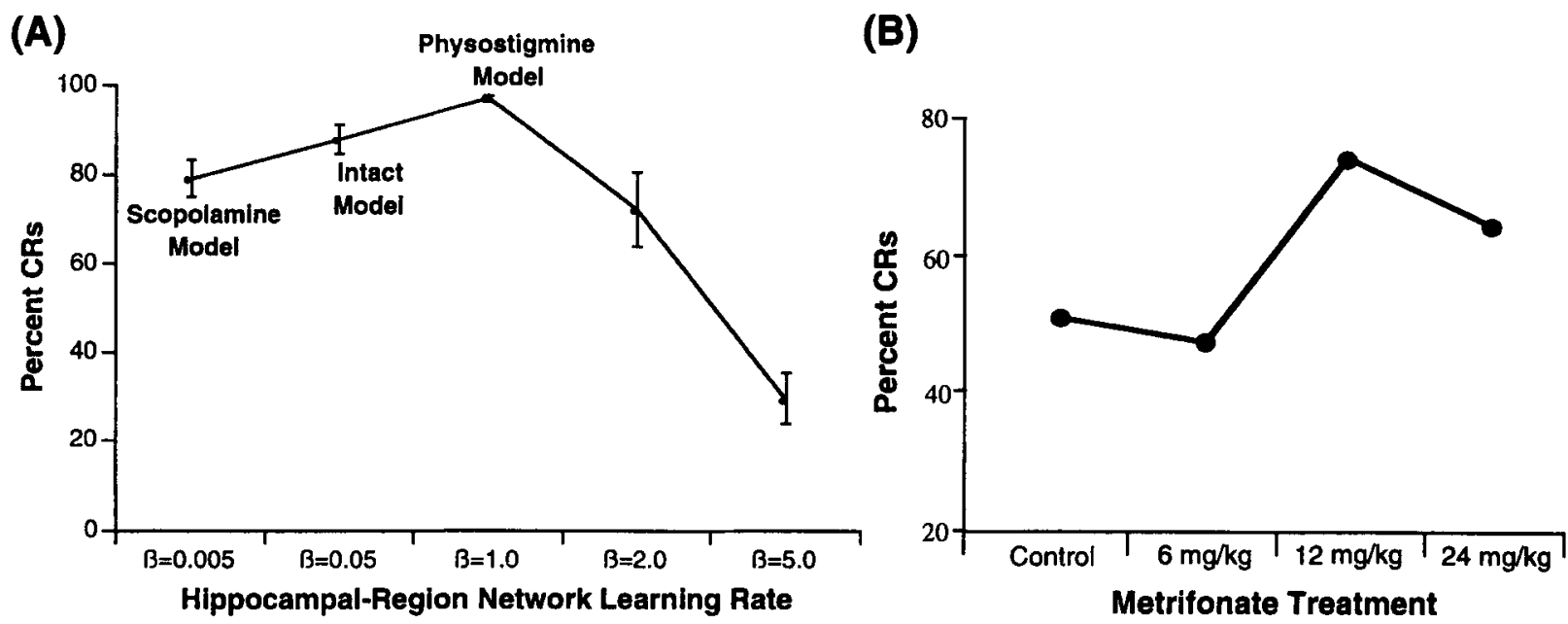

Figure 5. (A) Effect of hippocampal region network learning rates on learning speed in the model. Learning is faster as the learning rate increases from the scopolamine model $(\beta=0.005)$, through the intact model $(\beta=0.05)$, to a model of low doses of cholinergic agonists such as physostigmine $(\beta=1.0)$. However, further increases in the learning rate can result in no improvement $(\beta=2.0)$ or even degraded learning $(\beta=\mathbf{5 . 0})$, as the network becomes unstable. Thus, the model predicts a dose-dependent effect of cholinergic agonists on learning. (B) A recent study confirmed this prediction of a U-shaped dose response curve (Kronforst-Collins et al., 1997). Rabbit eyeblink conditioning was enhanced by a moderate dose of the cholinesterase inhibitor metrifonate $(12 \mathrm{mg} / \mathrm{kg}) \mathrm{more}$ than by a higher $(24 \mathrm{mg} / \mathrm{kg})$ or lower $(6 \mathrm{mg} / \mathrm{kg})$ dose. Figure plotted from data presented in Kronforst-Collins et al. (1997). 
information transfer between networks, the number of nodes in the networks, and so on. Although these have less immediate parallels to what is known about the substrate, they are computationally plausible.

One way to strengthen the case that the assumptions in the model are valid would be to show that they are sufficient to account for a wider range of behavioral data. The goal of this paper is, accordingly, to test the model more fully, by applying it to a range of data regarding conditioning under scopolamine and related drugs. To the extent that the model generates correct performance, it is more likely that the model assumptions are valid. The paper also considers several effects that do not involve eyeblink conditioning, and therefore are beyond the direct scope of the model, but that can still be discussed in terms of this general approach to understanding septohippocampal interaction.

There are several limitations of the model that should be clearly delineated at the start.

1. The model is only compared with data from the classical eyeblink conditioning preparation. The cerebellar network in the model is a simplification of Thompson's (1986) description of the neurobiological circuitry underlying eyeblink conditioning; both these substrates and the behavior itself appear to be consistent across species, including rabbits and humans (see Woodruff-Pak, Li, \& Kem, 1994).

Other learning preparations depend on different neurobiological substrates; as a result, they may be more or less susceptible to interference by scopolamine, depending on the number and sensitivity of the muscarinic receptors involved in those substrates (see Izquierdo, 1989). So, although antimuscarinics have been shown to disrupt a wide range of learning preparations (see Spencer \& Lal, 1983), a particular dosage of scopolamine that is sufficient to disrupt learning in a sensitive substrate may not suffice to disrupt learning in a less sensitive substrate. For example, a low dose of systemic scopolamine can spare acquisition but disrupt reversal of a brightness discrimination in rats (Soffie \& Lamberty, 1987); a dose of systemic scopolamine that spares fear conditioning to a tone CS can disrupt the collateral conditioning to the experimental context (Anagnostaras, Maren, \& Fanselow, 1995). Perhaps not coincidentally, both reversal learning and contextual learning have been frequently associated with the hippocampus (see, e.g., Hirsh, 1974; Myers \& Gluck, 1994); these results therefore suggest that the septohippocampal system may be especially sensitive to systemic scopolamine (see also Anagnostaras et al., 1995).

Further, the choice of injection site clearly affects different preparations differently. For example, scopolamine injected directly into the medial septum impairs eyeblink conditioning but not heartrate conditioning (Powell, Hernandez, \& Buchanan, 1985)--probably only reflecting the fact that heartrate conditioning does not depend on the medial septum (Powell, Milligan, \& Mull, 1982) - whereas medial septal lesions, which disrupt septohippocampal cholinergic pathways, devastate eyeblink conditioning (Berry \& Thompson, 1979).
For these reasons, it is worth exercising caution when generalizing across preparations to summarize the effects of scopolamine. The current model focuses on the effects of scopolamine on eyeblink conditioning. Studies from other preparations can be discussed-and even qualitatively addressed by the model--but it should always be emphasized that these preparations may show different degrees of scopolamine-mediated impairment.

2. The model accounts for data on the effects of scopolamine in associative learning; the model does not claim to capture any nonassociative or peripheral effects of scopolamine treatment. For example, as well as disrupting learning, scopolamine has anesthetic properties and is often used to prevent motion sickness (see Feldman \& Quenzer, 1984, p. 141, for a review of therapeutic uses). These additional drug actions are not reflected in the model, although it is possible that they may contribute to the deleterious effects of the drug on expressing learning. It is worth noting, however, that many empirical studies consider a comparison group given methylscopolamine, a form of scopolamine that does not cross the bloodbrain barrier, and thus can be used to rule out any peripheral explanations for altered learning performance.

3. The model, in its current form, addresses only trial level data, meaning that it does not capture any real-time effects, such as the form of the conditioned response or the effects of manipulating stimulus scheduling within a trial. For example, one study suggests that scopolamine may have a proportionately greater effect on eyeblink conditioning when there is a short delay between CS and US (trace conditioning) than when CS and US coterminate (delay conditioning; Kaneko \& Thompson, 1997). The current model cannot address these results, although the addition of recurrent connections may suffice to allow the model to address many real-time aspects of conditioning, including the differences between trace and delay conditioning (Zackheim, Myers, \& Gluck, 1997).

4. Finally, the model is designed to focus on adaptive representations in the hippocampal region and their effect on behavior. The output of the model is interpreted as affecting the strength of or the probability that a behavioral response is generated by motor effectors, but it is not itself identical to that behavioral response. Therefore, neither the absolute value of the model output nor the absolute number of trials for achieving a particular performance criteria can be directly compared against empirical data. The appropriate level of comparison between model and subject performance is qualitative, in terms of how manipulations to septohippocampal processes alter performance relative to control conditions.

These limitations do not represent failures of the model per se but rather describe its boundaries-what it is intended to achieve - and should be kept in mind while evaluating the model's degree of success and utility. Full details of the simulation parameters are given in the appendix.

\section{Learning Recovers After Scopolamine Removed}

As discussed above, the most general finding regarding scopolamine is that it slows conditioned acquisition, 

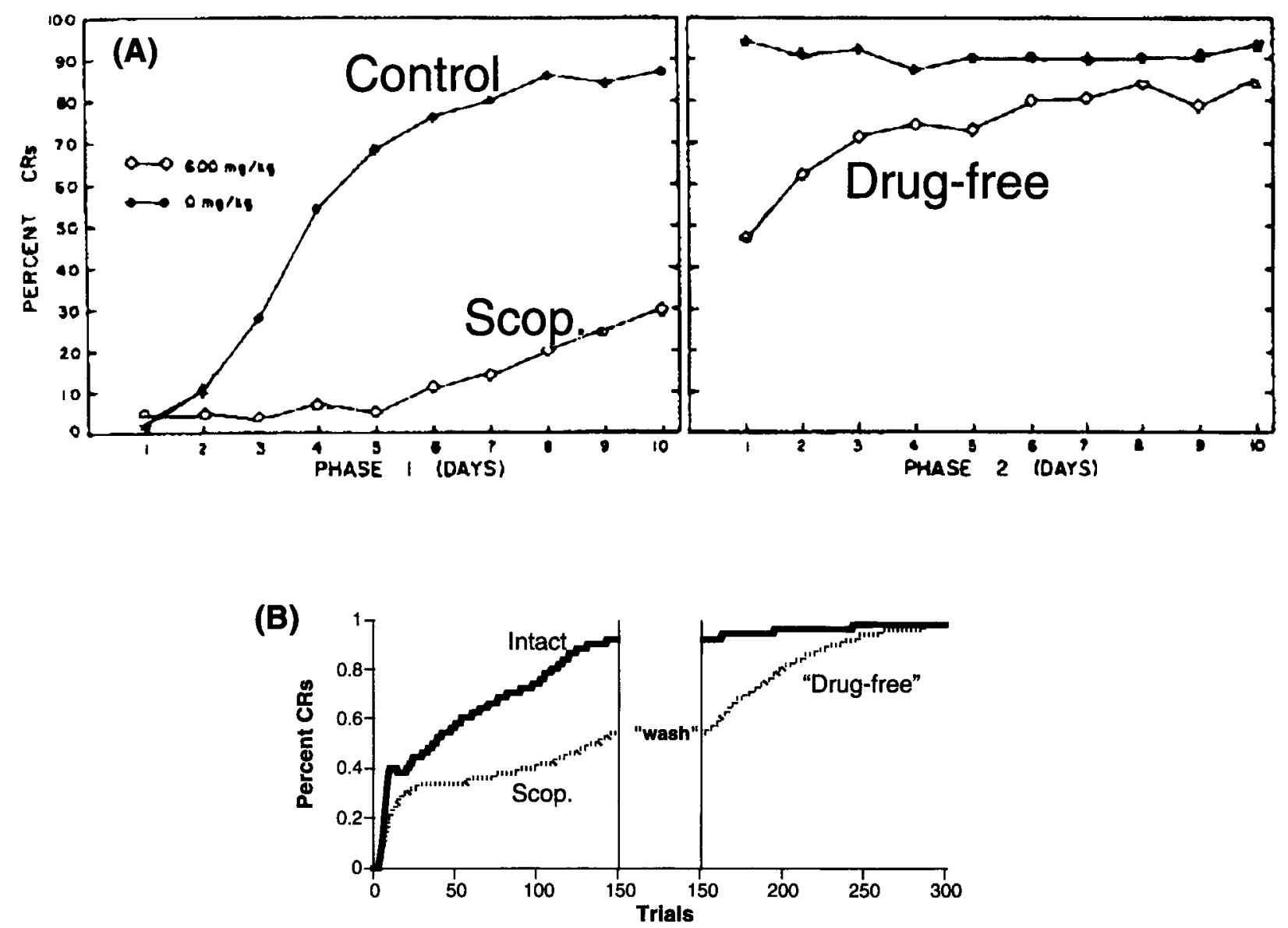

Figure 6. (A) Harvey et al. (1983) showed that control rabbits could learn a conditioned stimulus-unconditioned stimulus (CS-US) association to asymptote within about 10 days of training. Rabbits given equal training under systemic scopolamine were impaired. The scopolamine rabbits were then allowed to recover from the effects of the drug and given 10 additional days of drug-free training. These animals showed an initial performance decrement but then quickly went on to reach the same asymptotic performance levels as controls. (B) The scopolamine model shows a similar effect in Phase 1, learning a CS-US association more slowly than did the intact model. In Phase 2, the scopolamine model is returned to normal (intact) hippocampal region network learning rates to simulate a drug-free phase, and learning proceeds quickly to normal asymptote. From "Effects of Scopolamine and Methylscopolamine on Classical Conditioning of the Rabbit Nictitating Membrane Response," by J. Harvey, I. Gormezano, and V. Cool-Hauser, 1993, Journal of Pharmacology \& Experimental Therapeutics, 225, p. 45, Figures 1A-1B. Copyright 1983 by The American Society for Pharmacology and Experimental Therapeutics. Adapted with permission.

specifically by delaying the onset of conditioned responding. Harvey, Gormezano, and Cool-Hauser (1983) considered animals given several days of eyeblink conditioning under scopolamine and demonstrated the usual retarded learning. These animals were then allowed to recover from the drug effects for (drug-free) training. Initially, the animals continued to show reduced responding, indicating that scopolamine had retarded acquisition and not merely performance. However, the animals then went on to acquire the conditioned response quickly and reached the same asymptotic performance levels as a control group (Figure 6A).

The model can be subjected to the same procedure: A scopolamine model is given $150 \mathrm{CS}-\mathrm{US}$ pairings and shows retarded learning relative to a control intact model given identical training (Figure 6B). ${ }^{\prime}$ The hippocampal region network learning rate in the scopolamine model is then returned to normal levels for 150 more CS-US pairings. At first, responding stays low, since it takes some time for the hippocampal region network to develop new representations and transfer these to the cerebellar network. Thus, the rate of responding on Trials 151-160 in the scopolamine model is significantly slower than in the control intact model $[t(38)=4.005, p<.005]$. However, once the hippocampal region network develops its representations and these are acquired by the cerebellar network, learning proceeds quickly, within about 100 additional trials, and reaches the same asymptote as in the control simulations.

\section{Latent Inhibition Is Preserved Under Scopolamine}

In normal animals, CS--US learning is slowed after unreinforced exposure to the CS-as compared with ani- 

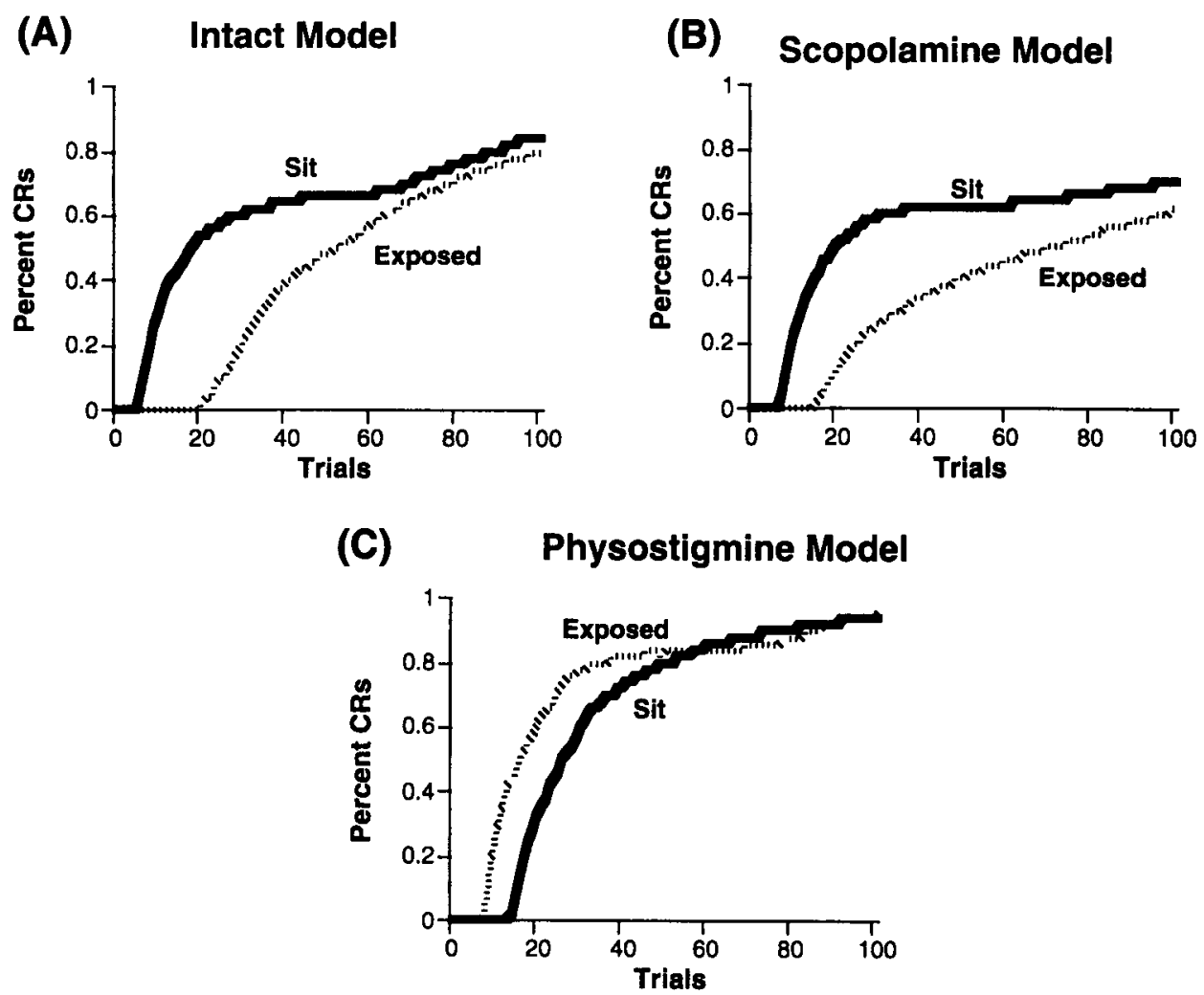

Figure 7. (A) Latent inhibition in the intact model. Learning a conditioned stimulus-unconditioned stimulus (CS-US) association is slower in simulations that first received 150 CS-alone trials (exposed group), as compared with simulations that receive 150 context-alone trials (sit control group). This effect results from hippocampal-region-mediated compression of context and CS during the exposure phase, slowing later learning to respond to the CS but not context alone. (B) Latent inhibition in the scopolamine model. Learning of a CS-US association is slow in the sit group, as compared with the intact model, but the exposed group is slower still. This latent inhibition effect obtains because the hippocampal region network is slowed but not disabled in the scopolamine model, and so compressed representations are still formed over the course of the 150 exposure trials. This preservation of latent inhibition appears consistent with animal findings (Moore et al., 1976). (C) Latent inhibition in the physostigmine model. With a high enough hippocampal region learning rate (i.e., $\beta=1.0), C S-U S$ learning is at a ceiling, and the effects of CS exposure are not visible. A similar de facto elimination of latent inhibition is expected in eyeblink conditioning with high doses of cholinergic agonists.

mals who received an equal amount of exposure to the context alone (referred to as sit controls). This effect is termed latent inhibition (Lubow, 1973). The intact model correctly shows this effect: Simulations which receive $150 \mathrm{CS}$-alone exposures are subsequently slower to learn the CS-US association than are sit controls that receive 150 trials with the context alone (Figure 7A). This latent inhibition effect depends on hippocampal region mediation in the intact model: During the exposure phase, the $\mathrm{CS}$ and context cooccur and are both equally unpredictive of any salient events (such as the US), and so their representations are compressed. This slows later learning to respond to the CS but not to the context alone (Myers \& Gluck, 1994). Since this hippocampal region mediation is absent in the lesioned model, there is no latent inhibition in the lesioned model (Myers \& Gluck, 1994); this is consistent with data showing that broad hippocampal region damage also disrupts the effect (Kaye \& Pearce, 1987; Solomon \& Moore, 1975).

Systemic scopolamine reduces but does not eliminate latent inhibition in eyeblink conditioning: Although learning is slower under scopolamine, exposure to the CS has been shown to retard that learning still further (Moore et al., 1976). The scopolamine model shows a similar effect: Although scopolamine is assumed to retard hippocampal region learning and hence to delay onset of responding, it does not completely disable the hippocampal region. Therefore, over the course of an exposure period, some representational compression will take place in the hippocampal region network, even under scopolamine. Thus, in the scopolamine model, CS-US learning is slow, but CS exposure slows CS-US learning still further, resulting in a latent inhibition effect (Figure 7B). The differences between exposed and sit groups and between in- 
tact and scopolamine groups are both significant in terms of trials to reach criterion in CS-US learning $[F(36)>$ $4.5, p<.05]$, with no significant interaction.

In contrast to the effects of muscarinic antagonism, the model predicts that muscarinic agonists should speed hippocampal region learning rates. This will be true whether or not CS exposure has occurred, and so, in principle, latent inhibition should not be affected: Both exposed and nonexposed groups will learn quickly, but the nonexposed group should learn more quickly than the exposed group. However, a moderate dose of such an agonist may speed CS-US learning to the point where there is a ceiling effect and no difference between groups is evident. Figure 7C shows this behavior in a cholinergic agonist model, where the hippocampal region learning rate is set higher than its normal value. No latent inhibition effect is evident: There is no significant difference in trials to criterion for exposed as opposed to nonexposed conditions $[t(38)=0.68, p>.5]$. At a still higher dose, cholinergic agonists would be expected to result in an unstable hippocampal region network that would prevent CS-US learning in either the exposed or the nonexposed group.

The effects of direct or indirect cholinergic agonists on latent inhibition in eyeblink conditioning remain to be investigated. In rat conditioned emotional responding, the cholinergic agonist nicotine disrupts latent inhibition (Joseph, Peters, \& Gray, 1993). We have already noted the caution necessary in generalizing across results from different preparations. Additionally, nicotine acts at a subset of cholinergic receptors that are distributed quite differently from the muscarinic cholinergic receptors blocked by scopolamine. Thus, nicotine may have quite different effects than a muscarinic agonist.

There have also been recent studies evaluating latent inhibition after a selective immunotoxic lesion of the cholinergic projections from the basal forebrain to the hippocampus. Unfortunately, the results are mixed: The

\section{(A) Intact Model}

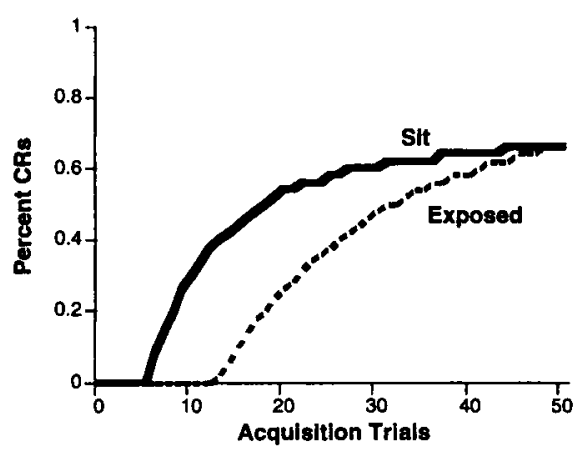

(B) Intact Model, Phase 1 Representations

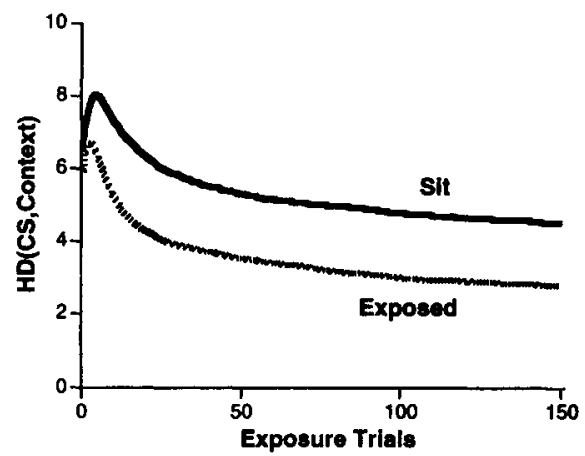

(C) Scopolamine Model

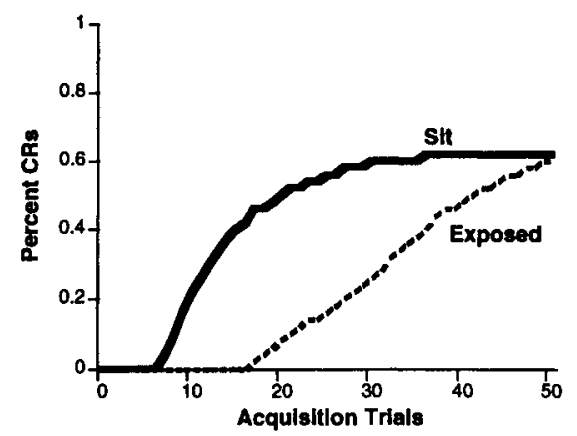

Figure 8. (A) The intact model shows learned irrelevance: Conditioned stimulus-unconditioned stimulus (CS-US) learning is slowed after uncorrelated exposure to CS and US, as compared with a control group that is exposed to the context alone. (B) This learned irrelevance effect is a result of representational compression during Phase 1, in which the CS and context are equally nonpredictive of the US. The representations of CS and context grow more similar, as reflected in a reduced $\mathrm{HD}(\mathrm{CS}$,context) - that is, the Hamming distance between activation patterns in the hippocampal region's internal layer nodes to the CS versus context alone. HD(CS,context) is much lower in the exposed simulations than in sit controls. (C) The scopolamine model also shows learned irrelevance. For direct comparison with the Harvey et al. (1983) data, the model is given uncorrelated CS/US exposure under scopolamine conditions, followed by CS-US learning under intact conditions. CS-US exposure slows learning relative to sit controls. 
immunotoxic lesion eliminates latent inhibition in rat appetitive conditioning (Baxter, Holland, \& Gallagher, 1997) but not in conditioned taste aversion (Dougherty, Salat, \& Walsh, 1996). The effects of neurotoxic cholinergic lesion on latent inhibition remain to be investigated in rabbit eyeblink conditioning.

\section{Learned Irrelevance Is Preserved Under Scopolamine}

Learned irrelevance is a related effect in which the exposure phase contains uncorrelated presentations of both CS and US; subsequent CS-US learning is typically retarded far more than it is after exposure to CS alone or after exposure to US alone (Bennett, Maldonado, \& Mackintosh, 1995; see also Mackintosh, 1973). Further, there is evidence that learned irrelevance is not reducible to a sum of CS- and US-exposure effects but that the lack of correlation between CS and US is specifically responsible for retarding later learning of a predictive relationship between them (Baker \& Mackintosh, 1979; Bennet et al., 1995; Matzel, Schachtman, \& Miller, 1988).

This kind of predictive relationship is exactly what governs how new representations are formed in the intact model's hippocampal region network. As a result, the intact model shows learned irrelevance: Uncorrelated CS-US exposure retards CS-US learning relative to sit controls (Figure 8A). The exposed group averages 63.7 trials to criterion, whereas the sit control group averages only 37.2 trials to criterion $[t(17)=2.009, p<.05]$. The learned irrelevance effect obtains because of representational compression during the exposure phase. This compression can be quantified with a Hamming distance (HD) metric. This involves recording the response of each hippocampal region network internal layer node $i$ to the $\mathrm{CS}, y_{i}^{\mathrm{CS}}$, and to the context alone, $y_{i}^{c \times t}$, and then taking the difference between them, $d_{i}=\left|y_{i}^{\mathrm{CS}}-y_{i}^{\mathrm{cxt}}\right|$. If the two patterns evoke the same response, $d_{i}=0$; if they evoke maximally different responses, $d_{i}=1$. The $\mathrm{HD}(\mathrm{CS}, \mathrm{cxt})$ is then the sum of the $d_{i}$ over all nodes $i$. Early in training, $\operatorname{HD}(C S, c x t)$ should be some random value reflecting the network's initial representations of the CS and the context. Figure $8 \mathrm{~B}$ shows that $\mathrm{HD}(\mathrm{CS}, \mathrm{cxt})$ starts out at approximately the same level for the exposed and the sit control simulations. With CS-US exposure, the probability of the US is the same in the presence of the CS as in the absence of the CS. This means that both the context and the CS are equally (non)predictive of the US. Accordingly, the representations of CS and context are compressed together in the exposed simulations, and $\mathrm{HD}(\mathrm{CS}, \mathrm{cxt}) \mathrm{de}-$ creases to a level well below that in sit controls. This compression hinders subsequent discrimination of the CS and context, slowing learning as shown in Figure 8A.

Systemic scopolamine does not disrupt learned irrelevance in rabbit eyeblink conditioning (Harvey et al., 1983). Rabbits given scopolamine during uncorrelated CS-US exposure and then trained without the drug learn the CS-US association at the same speed as a group given similar exposure without the drug. Although Har- vey et al.'s (1983) study did not include direct comparison with a sit control group, the authors report that both of the exposed groups (normal and scopolamine) learn significantly more slowly than do a group of normal rabbits given no exposure.

To simulate the animal experiment, one group of scopolamine simulations is given uncorrelated CS-US exposure, whereas a second group of scopolamine simulations is treated as sit controls and given exposure to the context alone. All simulations are then returned to normal intact hippocampal region learning rates-simulating a drug-free phase-and given CS-US training. Figure $8 \mathrm{C}$ shows that the exposed simulations learn more slowly; thus, learned irrelevance is preserved under scopolamine in the model. In direct agreement with the Harvey et al. (1983) data, the exposed intact and scopolamine models learn at equal rates $[t(18)=0.716, p>.1]$.

In the scopolamine model, learned irrelevance obtains for the same reasons as latent inhibition-namely, although scopolamine is assumed to slow the formation of hippocampal-mediated representations in the exposure phase, it does not eliminate them. By the end of the exposure phase, the scopolamine model's hippocampal region network has formed representations that reflect the lack of correlation between CS and US, and these representations impede subsequent CS-US associations.

\section{Scopolamine Does Not Slow Conditioned Acquisition After Hippocampal Lesion}

Since the effect of systemic scopolannine on eyeblink conditioning is assumed to be disruption of hippocampal region processing, it might be expected that scopolamine would have little or no effect on animals with hippocampal region damage. As noted above, such lesions do not interfere with acquisition of a simple conditioned eyeblink response in animals, humans, or the model (refer to Figure 2). Systemic scopolamine administered to hippocampal-lesioned animals does not cause an impairment either (Figure 9A; Solomon et al., 1983). Since the
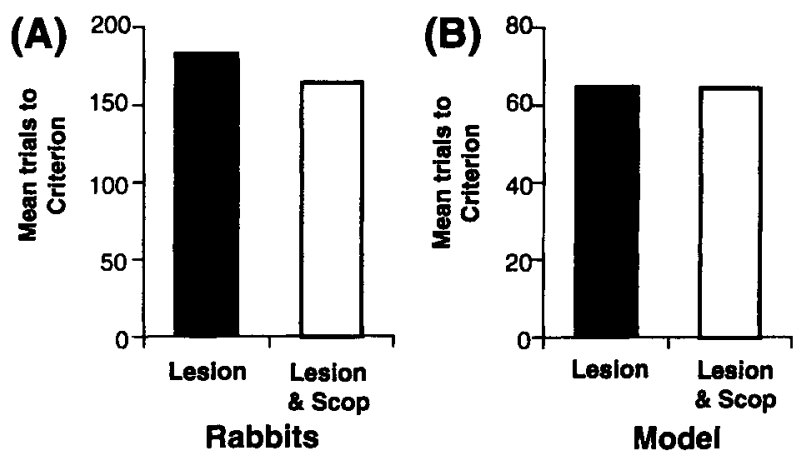

Figure 9. (A) Scopolamine does not retard eyeblink conditioning in rabbits with hippocampal damage (HL). Plotted from data presented in Solomon et al. (1983). (B) Since the scopolamine model involves reduced hippocampal region network learning rates, it is also trivially true that scopolamine does not affect learning in the lesioned model. 

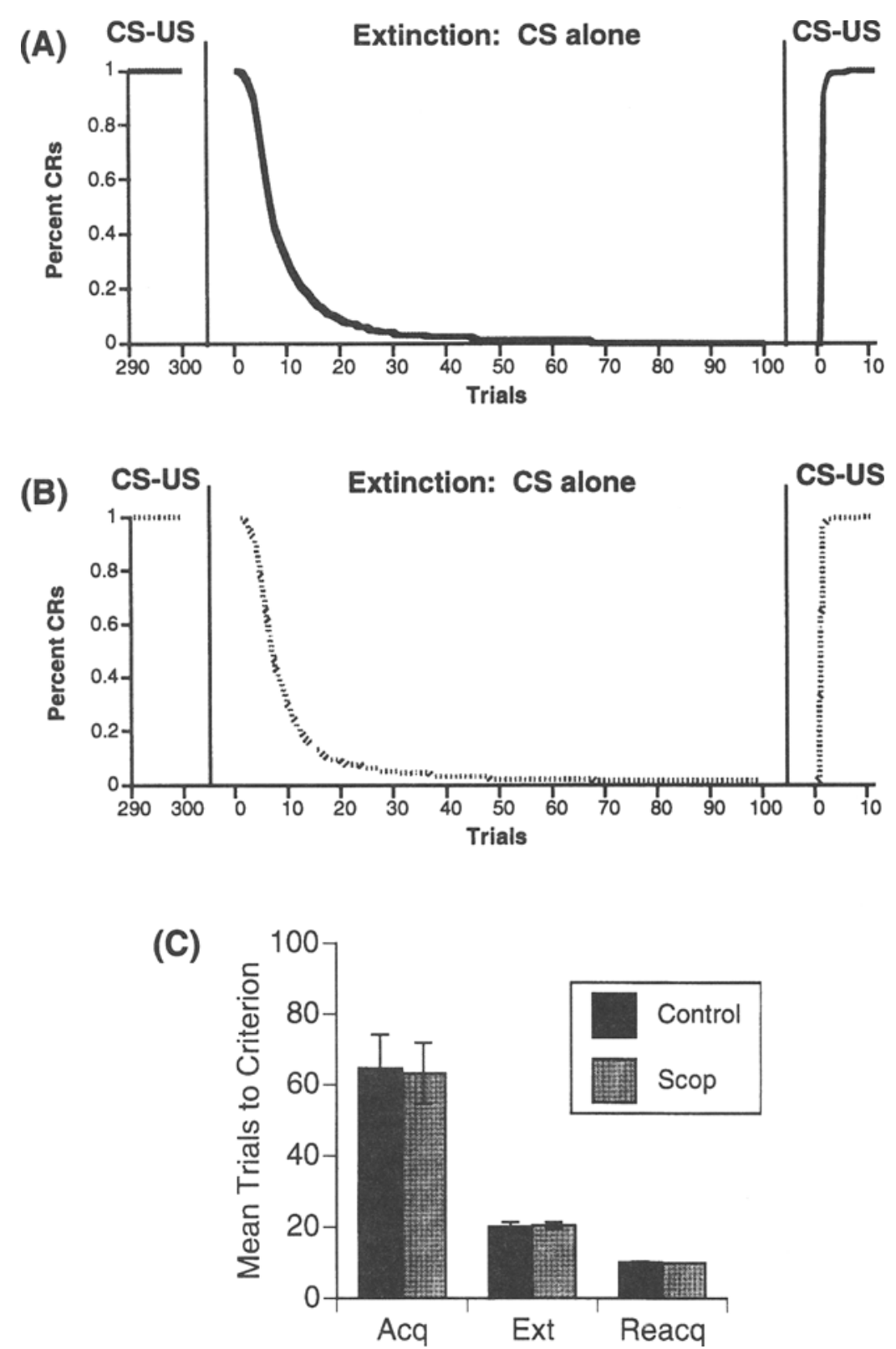

Figure 10. Extinction and reacquisition in the model. (A) After 300 trials associating conditioned stimulus (CS) and unconditioned stimulus (US), followed by CS-alone training, the intact model quickly extinguishes responding. During subsequent reacquisition training, pairing CS and US, the intact model quickly reinstates responding. (B) Following Scavio et al. (1992), the model is given "scopolamine" during the extinction phase (though not during acquisition or reacquisition phases); the model still extinguishes and reacquires normally. (C) In terms of trials to criterion, both the intact and scopolamine models extinguish at the same speed and reacquire the response more quickly than it was originally acquired.

model assumes that scopolamine serves to reduce hippocampal region learning rates and since the hippocampal region network is disabled in the lesioned model, it is also trivially true that there is no disruption of acquisition in the lesioned model under scopolamine (Figure 9B).

\section{Scopolamine Does Not Disrupt Extinction}

A response learned to a CS can be extinguished by subsequent training with the CS alone and no US. In animals, the CR gradually weakens and finally disappears. If this is followed by another phase of CS-US training, the $\mathrm{CR}$ is reacquired, typically much faster than it was 
originally acquired (see, e.g., Gormezano et al., 1983). This implies that extinction is not merely the unlearning of the original response but rather the suppressing of it or the setting up a CS-no-US association that competes with the earlier CS-US association; thus, extinction trials do not erase the original learning, which can be quickly reinstated during acquisition training.

Figure 10A shows that the intact model also shows rapid reacquisition of an extinguished CR. During the original acquisition session, the hippocampal region network develops new stimulus representations; these are acquired by the cerebellar network, which then maps from them to the CR. Then, during the extinction session, the CS is no longer paired with a US, and error results when the model generates a CR. The cerebellar network quickly learns to map from the stimulus representation to no-CR- extinguishing the response. This happens within a few trials, long before any substantive change to the stimulus representations in the internal layer of either the hippocampal region or the cerebellar network. As a result, those same stimulus representations are readily available during the third (reacquisition) phase, when the CS and US are again paired and a CR quickly reemerges.

This explanation of extinction is broadly consistent with some empirical data, although there are other data suggesting that extinction represents the development of an explicit association between CS and no-US, which competes with the earlier CS-US association (see, e.g., Bouton, 1991). Currently, it is still a matter of much debate as to what processes are actually invoked during extinction in the brain.

The model explanation assumes that hippocampalrepresentational changes occur during acquisition but are not particularly modified during extinction and reacquisition. Accordingly, the model expects that there should be little effect of hippocampal disruption through scopolamine during extinction. Scavio, Clift, and Wills

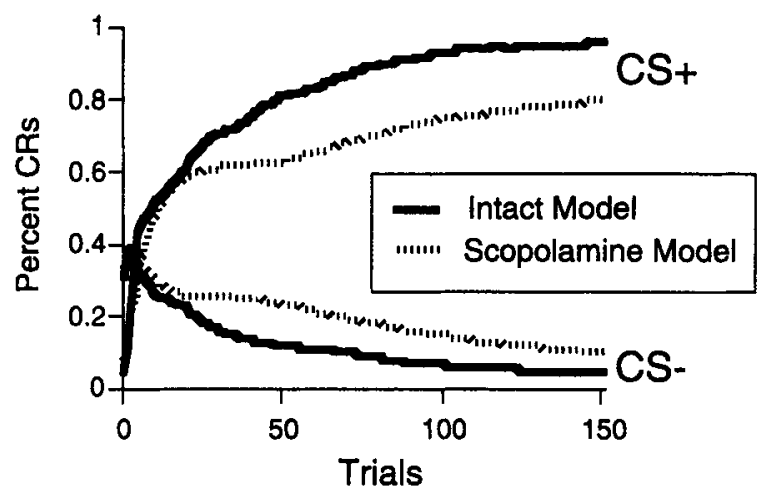

Figure 11. The scopolamine model shows impaired discrimination, learning te generate a conditioned response (CR) to one conditioned stimulus $(\mathrm{CS}+$ ) but not to a second $\mathrm{CS}-$. The effects of scopolamine on rabbit eyeblink discrimination remain to be determined experimentally, although discrimination is impaired following septal lesion or systemic administration of the muscarinic agonist atropine.
(1992) in fact demonstrated that animals trained to give eyeblink CRs would extinguish this responding at the same rate with and without scopolamine; animals extinguished under scopolamine later reacquired the response at the same rate as did animals extinguished without the drug. Figure 10B shows the same behavior in the model, and Figure $10 \mathrm{C}$ confirms that both the intact and the scopolamine models extinguish at the same speed and reacquire much more quickly than they learned the original association.

\section{Conditioned Discrimination: \\ Mixed Empirical Results}

Whereas acquisition involves learning to respond to a single stimulus, conditioned discrimination involves learning to respond to one stimulus (CS+) but to withhold responding to a second stimulus (CS-). The stimuli are often two highly distinguishable tones. Hippocampal lesion does not impair discrimination learning in rabbit eyeblink conditioning (see, e.g., Berger \& Orr, 1983; Schmaltz \& Theios, 1972). However, in the same way that scopolamine disrupts acquisition - even though hippocampal lesion does not-it might be expected to disrupt discrimination learning.

This is just the behavior shown by the model (Figure 11): Whereas the intact model quickly learns to respond to $\mathrm{CS}+$ but not to $\mathrm{CS}-$, the scopolamine model takes longer to discriminate the stimuli. In the intact model, the hippocampal region network constructs new representations that differentiate predictive stimulinamely, CS+ and CS - . This facilitates mapping them to different responses, which leads to fast learning. In the scopolamine model, the hippocampal region network takes longer to accomplish this differentiation, making it harder for the cerebellar network to map CS+ and CSto opposite responses. The form of the disruption is, therefore, evident as less consistent responding to CS+ and more erroneous responding to $\mathrm{CS}-$. Eventually, as with acquisition, the scopolamine model reaches normal levels of performance.

Unfortunately, the empirical results on this issue are mixed. One unpublished study reported no effects of scopolamine on eyeblink discrimination (see Moore et al., 1976); however, systemic atropine, another muscarinic blocker, does disrupt discrimination (Downs et al., 1972), as does septal lesion (Lockhart \& Moore, 1975; Powell et al., 1976). Even these results are contradictory, however, since the effect of atropine is to disrupt discrimination by producing overresponding to the unreinforced CS-, whereas septal lesion produces underresponding to the reinforced CS+. Further empirical studies clearly are needed to determine why scopolamine, atropine, and septal lesion might have different effects on discrimination learning.

One likely issue is that scopolamine has a higher receptor binding affinity than does atropine (see, e.g., Feldman \& Quenzer, 1984, p. 141), and so larger doses of atropine are typically needed to produce the sort of re- 
tarded acquisition curves seen under scopolamine-for example, whereas a typical scopolamine study might use a dosage of $1.5 \mathrm{mg} / \mathrm{kg}$ (Moore et al., 1976; Salvatierra \& Berry, 1989; Solomon et al., 1983), the Downs et al. (1972) study used atropine dosages ranging from 10 to $26 \mathrm{mg} / \mathrm{kg}$. Such a high dosage of a cholinergic antagonist might be expected to produce a broader range of effects. Peripheral systems are probably not implicated, since, at the dosages reported, atropine did not interfere with unconditioned reflex responding, which implies that the learning impairment did not simply result from an inability to produce blinks. However, the cerebellum plays a critical role in eyeblink conditioning (Lewis, LoTurco, \& Solomon, 1987; Thompson, 1986), and there are cholinergic receptors in the cerebellum (see, e.g., Krnjevic, 1975). Systemic administration of a high dose of atropine may therefore well interrupt the ability of cerebellum to express conditioned eyeblink responses. Consistent with this suggestion, atropine does suppress production of well-learned conditioned eyeblink responding; once the drug is removed, responding rebounds to normal levels (Downs et al., 1972). In contrast, expression of welllearned conditioned responses is not prevented in the scopolamine model or in animals given scopolamine (Scavio et al., 1992).

Interference with conditioned response expression would not seem to be such an issue after medial septal lesion, since the primary cholinergic afferents to the cerebellum arise not from the medial septum but from the pons and midbrain tegmentum (see, e.g., Barmack, Baughman, \& Eckenstein, 1992). Therefore, the retardation of discrimination learning after medial septal lesion is more likely to reflect a truly associative deficit. Since septal damage interrupts both cholinergic and noncholinergic hippocampal afferents (see below), it cannot be definitively inferred that scopolamine would have a similar effect. At this point, pending further empirical studies, the only conclusion that can be drawn with any certainty is that septohippocampal cholinergic processes are not necessary for eventual acquisition of a conditioned discrimination, whereas the model predicts that disrupting these processes through scopolamine should nonetheless suffice to slow acquisition of such a discrimination.

\section{LOCALIZED SCOPOLAMINE STUDIES}

The previous section presented several new simulation results, in which the model was applied to eyeblink conditioning paradigms and shown to account for the effects of systemic scopolamine on learning. Much of the effect of systemic scopolamine may be due to blockade of postsynaptic cholinergic receptors at terminals of the septohippocampal pathway. However, septal neurons also contain cholinergic receptors (Solomon \& Gottfried, 1981). Thus, injections of scopolamine directly into the medial septum may affect firing of cholinergic neurons there, which, in turn, project to hippocampus (Mosko et al., 1973). Such medial septal scopolamine injections do in fact disrupt conditioning in much the same way as does systemic scopolamine (Powell et al., 1985; Solomon \& Gottfried, 1981). Conversely, the lateral septum receives output from the hippocampus but does not send a strong input to the hippocampus (see, e.g., Toth, Borhegyi, \& Freund, 1993), and so scopolamine injected to the lateral septum would not be expected to particularly affect conditioned acquisition. Again, empirical studies show this to be the case (Powell et al., 1985).

One curious result, which may at first glance appear contradictory to the current hypothesis, is the finding that scopolamine injected directly to the dorsal hippocampus does not disrupt eyeblink conditioning in rabbits (Solomon \& Gottfried, 1981). This seems to suggest that septohippocampal cholinergic inputs are not necessary for conditioned learning.

There are several possible explanations for this finding that do not contradict the current hypothesis. First, there is the possibility that Solomon and Gottfried's (1981) injections to the dorsal hippocampus left enough acetylcholine available in the ventral hippocampus to sustain learning. Most of the cholinergic septohippocampal projection targets hippocampal field CA1 (Krnjevic \& Ropert, 1982), of which large portions are ventral in rabbits and therefore may have been spared in Solomon and Gottfried's study. Additionally, although most acetylcholine (up to $90 \%$ in rats) enters the hippocampus through the fornix into the dorsal hippocampus, there is also a ventral pathway, which is even more significant in higher species (Zola-Morgan, Squire, \& Amaral, 1989); damage limited to the dorsal hippocampus would presumably spare this input. However, it is not clear whether this ventral pathway includes septal input, and its activity may be correlated with very different processes (such as general arousal) than those considered here.

A second possibility is that, although most of the cholinergic receptors in the hippocampus are muscarinic (Spencer \& Lal, 1983) and thus subject to blockade by intrahippocampal scopolamine, there are also nicotinic receptors in the hippocampus that would not be so affected (see Woodruff-Pak et al., 1994); it is possible that disruption to both nicotinic and muscarinic receptors in the hippocampus is necessary to retard learning (see also Woodruff-Pak et al., 1994, for a discussion of the importance of brain nicotinic receptors in learning). Systemic scopolamine, by contrast, presumably reduces the amount of acetylcholine released by the medial septum and would, in turn, reduce the amount of acetylcholine activating both muscarinic and nicotinic receptors in the hippocampus, causing disrupted learning.

A third possibility was suggested by Solomon and Gottfried (1981): that the critical locus of scopolamine in disrupting hippocampal processing is not in the hippocampus at all but presynaptically in the medial septum, blocking septal autoreceptors and/or noncholinergic septal neurons. GABAergic cells in the medial septum, which target hippocampal interneurons, may modulate theta rhythm, a hippocampal EEG rhythm associated with ex- 
ploratory behaviors (Berry \& Thompson, 1979; Buzsáki \& Eidelberg, 1983). The rhythmic (4-8 Hz) bursting of theta rhythm alternates with periods of sharp wave activity, characterized by nonbursting activity (Fox, Wolfson, \& Ranck, 1983), which occur during consummatory behaviors such as grooming and eating (Vanderwolf \& Leung, 1983). Buzsáki (1989) has suggested that this alternation between theta and sharp wave states corresponds to two phases of hippocampal system processing: theta representing a storage phase, in which incoming information is quickly stored in the hippocampal system, and sharp waves representing a consolidation phase, during which stored hippocampal memories are reinstated for gradual transfer to permanent neocortical storage.

If these GABAergic processes are disrupted by scopolamine, that would be another way in which learning might be disrupted. In fact, systemic scopolamine does disrupt hippocampal theta (Salvatierra \& Berry, 1989; Stumpf, Petsche, \& Golgolak, 1962), but there is some evidence that hippocampal theta rhythm can survive total disruption of the septohippocampal cholinergic system (Lee, Chrobak, Sik, Wiley, \& Buzsáki, 1994). This would imply that the effect of scopolamine on learning cannot wholly be attributed to indirectly disrupting GABAergic septohippocampal processes.

In fact, it is entirely likely that hippocampal processing is modulated by both GABAergic and cholinergic processes. For example, Lee et al. (1994) hypothesize that, whereas septal GABAergic afferents are responsible for the rhythmic firing of hippocampal interneurons that underlies the theta rhythm, the cholinergic projection may serve to increase the population phase-locking of cells and thereby regulate the magnitude of theta (see also Brazhnik et al., 1993). Additionally, activation of both muscarinic acetylcholine receptors (Hasselmo \& Schnell, 1994) and GABA-B receptors (Wallenstein \& Hasselmo, 1997) could further enhance information storage by selectively suppressing recurrent collateral activity and increasing plasticity.

In terms of the current model, although septohippocampal GABA is not directly included, the qualitative idea is still that medial septal inputs (both cholinergic and GABAergic) would enhance hippocampal region storage and thus that drugs (such as scopolamine) that disrupt these inputs would reduce the rate of learning in the hippocampal region. Unfortunately, at present there is little empirical work detailing the effects of manipulating septohippocampal GABA in rabbit eyeblink conditioning, so these predictions remain to be fully tested. Further modeling work that includes a role both for cholinergic and for GABAergic septohippocampal processes in conditioning may help provide a more detailed framework for studying the interactions between these two septohippocampal projections.

\section{DISCUSSION}

This paper aimed to test the corticohippocampal model and its proposed incorporation of septohippocampal cho- linergic modulation against a range of empirical data. The model was originally shown (Myers et al., 1996) to correctly account for the dose-dependent effects of systemic scopolamine in disrupting classical eyeblink conditioning, specifically by prolonging the onset of learning. It also provides an explanation for the facilitation of learning with moderate doses of physostigmine but not larger doses. This paper showed that the model could correctly account for several additional effects within the same domain: namely, that scopolamine does not retard learning after hippocampal lesion, that fast learning resumes once scopolamine is removed, and that scopolamine appears to spare latent inhibition, learned irrelevance, and extinction. Finally, although the results regarding the effects of cholinergic disruption on discrimination learning are contradictory, the model shows a scopolamine deficit that may be consistent with the data. Although the model, as it currently stands, cannot directly address the effects of localized scopolamine, the underlying hypothesis - that scopolamine slows hippocampal region processing - is consistent with findings that conditioning is impaired after scopolamine injected to the medial septum, afferent to the hippocampus, but not to the lateral septum, efferent to the hippocampus. One curious result, that direct hippocampal scopolamine does not impair learning, may argue that the hippocampus is not the locus of cholinergic disruption, but there are several alternate possibilities that remain to be explored empirically.

The motivation for these simulations was to test the model-and hypothesis-as completely as possible against existing empirical data. As mentioned above, there are a number of parameters in the model that could have been manipulated to reduce acquisition learning in a manner broadly analogous to that seen in the scopolamine data. The chosen manipulation stands out among them in that it is related to prior behavioral and physiological hypotheses and demonstrates the ability to accurately generate behavioral effects across a range of conditioning paradigms.

The model does not account for the peripheral and nonassociative effects of scopolamine and, therefore, may not address all of the behavioral nuances seen under the drug. Additionally, it does not explicitly include any account of theta rhythm, which is believed to depend on GABAergic septohippocampal inputs (Berry \& Thompson, 1979; Buzsáki \& Eidelberg, 1983) and which is also disrupted by scopolamine. An important line of future modeling work will be to consider these GABAergic inputs and how they can combine with septohippocampal cholinergic inputs to provide finer control over the storage and recall processes in hippocampus (see also Wallenstein \& Hasselmo, 1997).

One of the major reasons for the interest in understanding cholinergic mechanisms in learning is the cholinergic depletion observed in neuropsychiatric diseases such as Alzheimer's dementia (Kesner, 1988; Whitehouse et al., 1982), which may underlie some of the cognitive syndromes observed in that disease (de Leon et al., 1993). Consistent with this explanation, some limited memory 
improvement can be observed in these patients after therapy with cholinergic agonists such as Tacrine (Knapp et al., 1994; Manning, 1994; Wagstaff \& McTavish, 1994) or physostigmine (Davis \& Mohs, 1982; Sevush, Guterman, \& Villalon, 1991; Thal, Fuld, Masur, \& Sharpless, 1983). Although a cure for this disease remains elusive, the possibility of even partial treatment with cholinergic therapy can best be explored if the mechanisms by which cholinergic processes influence learning are well understood. To this end, both computational models and further empirical studies may interact and inform each other, providing new and promising research directions.

\section{REFERENCES}

Akase, E., Alkon, D. L., \& Disterhoft, J. F. (1989). Hippocampal lesions impair memory of short-delay conditioned eyeblink in rabbits. Behavioral Neuroscience, 103, 935-943.

anagnostaras, S., Maren, S., \& Fanselow, M. (1995). Scopolamine selectively disrupts the acquisition of contextual fear conditioning in rats. Neurobiology of Learning \& Memory, 64, 191-194.

Bahro, M., SChreurs, B., Sunderland, T., \& Molchan, S. (1995). The effects of scopolamine, lorazepam, and glycopyrrolate on classical conditioning of the human eyeblink response. Psychopharmacology, 122, 395-400.

BaKer, A., \& Mackintosh, N. (1979). Preexposure to the CS alone, US alone, or CS and US uncorrelated: Latent inhibition, blocking by context or learned irrelevance? Learning \& Motivation, 10, 278-294.

BALDI, P., \& HoRNIK, K. (1989). Neural networks and principal component analysis: Learning from examples without local minima Neural Networks, 2, 53-58.

BarKaI, E., \& Hasselmo, M. (1994). Modulation of the input/output function of rat piriform cortex pyramidal cells. Journal of Neurophysiology, 72, 644-658

Barmack, N., Baughman, R., \& Eckenstein, F. (1992). Cholinergic innervation of the cerebellum of rat, rabbit, cat, and monkey as revealed by choline acetyltransferase activity and immunohistochemistry. Journal of Comparative Neurology, 317, 233-249.

Baxter, M., Holland, P., \& Gallagher, M. (1997). Disruption of decrements in conditioned stimulus processing by selective removal of hippocampal cholinergic input. Journal of Neuroscience, 17, 52305236.

Bennett, C., Maldonado, A., \& Mackintosh, N. (1995). Learned irrelevance is not the sum of exposure to CS and US. Quarterly Journal of Experimental Psychology, 48B, 117-128.

BERGER, T., \& ORR, W. (1983). Hippocampectomy selectively disrupts discrimination reversal learning of the rabbit nictitating membrane response. Behavioural Brain Research, $8,49-68$.

BERRY, S., \& THOMPSON, R. (1979). Medial septal lesions retard classical conditioning of the nictitating membrane response in rabbits. Science, 205, 209-211.

Bouton, M. (1991). Context and retrieval in extinction and in other examples of interference in simple associative learning. In L. Dachowski \& C. Flaherty (Eds.), Current topics in animal learning: Brain, emotion and cognition (pp. 25-52). Hillsdale, NJ: Erlbaum.

Brazhnik, E., Vinogradova, O., Stafekhina, V., \& Kitchigina, V. (1993). Acetylcholine, theta-rhythm and activity of hippocampal neurons in the rabbit: I. Spontaneous activity. Neuroscience, 53, 961-970.

BUZSÁKI, G. (1989). Two-stage model of memory-trace formation: A role for "noisy" brain states. Neuroscience, 31, 551-570.

BuZsÁKI, G., \& EjDEL berG, E. (1983). Phase relations of hippocampal projection cells and interneurons to theta activity in the anesthetized rat. Brain Research, 266, 334-339.

Camprell, B., Lytle, L., \& Fibiger, H. (1969). Ontogeny of adrenergic arousal and cholinergic inhibitory mechanisms in the rat. Science, $166,635-637$.
Daum, I., Channon, S., \& Canavan, A. (1989). Classical conditioning in patients with severe memory problems. Journal of Neurology, Neurosurgery \& Psychiatry, 52, 47-51.

DAVIS, K., \& MoHs, R. (1982). Enhancement of memory processes in Alzheimer's disease with multiple-dose intravenous physostigmine American Journal of Psychiatry, 139, 1421-1424.

de Leon, M., Golomb, J., George, A., Convit, A., Rusinek, H., Morys, J., Bobinski, M., de Santi, S., Tarshish, C., NarkieWICZ, O., \& WisNiEWsKI, H. (1993). Hippocampal formation atrophy: Prognostic significance for Alzheimer's disease. In B. Corain, K. Iqbal, M. Nicolini, B. Winblad, H. Wisniewski, \& P. Zatta (Eds.), Alzheimer's disease: Advances in clinical and brain research (pp. 3546). New York: Wiley.

Dougherty, K., Salat, D., \& Walsh, T. (1996). Intraseptal injection of the cholinergic immunotoxin 192 IgG-saporin fails to disrupt latent inhibition in a conditioned taste aversion paradigm. Brain Research, 736, 260-269.

Downs, D., Cardozo, C., Schneiderman, N., Yehle, A, VanderCAR, D., \& ZWILLING, G. (1972). Central effects of atropine upon aversive classical conditioning in rabbits. Psychopharmacologia, 23, 319-333.

Dumery, V., Derer, P., \& Blozovski, D. (1988). Enhancement of passive avoidance learning through small doses of intra-amygdaloid physostigmine in the young rat: Its relation to the development of acetylcholinesterase. Developmental Psychobiology, 21, 553-565.

EnNaCeur, A., \& Meliani, K. (1992). Effects of physostigmine and scopolamine on rats' performances in object-recognition and radialmaze tests. Psychopharmacology, 109, 321-330.

FELDMAN, R., \& QUENZER, L. (1984). Fundamentals of neuropsychology. Sunderland, MA: Sinauer.

Fox, S. E., WolfSon, S., \& Ranck, J. B., Jr. (1983). Investigating the mechanisms of hippocampal theta rhythms: Approaches and progress. In W. Seifert (Ed.), Neurobiology of the hippocampus (pp. 303-319). London: Academic Press.

Gabrieli, J., McGlinchey-Berroth, R., Carrillo, M., Gluck, M., Cermack, L., \& Disterhoft, J. (1995). Intact delay-eyeblink classical conditioning in amnesia. Behavioral Neuroscience, 109, 819-827.

GinN, S., \& PowELL, D. (1992). Nucleus basalis lesions attenuate acquisition, but not retention, of Pavlovian heart rate conditioning and have no effect on eyeblink conditioning. Experimental Brain Research, 89, 501-510.

GLUCK, M., \& Myers, C. (1993). Hippocampal mediation of stimulus representation: A computational theory. Hippocampus, 3, 491-516.

Gluck, M., Myers, C., \& Thompson, R. (1994). A computational model of the cerebellum and motor-reflex conditioning. In S. Zornetzer, J. Davis, C. Lau, \& T. McKenna (Eds.), An introduction to neural and electronic networks (pp. 91-98). New York: Academic Press.

Gluck, M., Oliver, L., \& MYERs, C. (1996). Late-training amnesic deficits in probabilistic category learning: A neurocomputational analysis. Learning \& Memory, 3, 326-340.

Gormezano, I., Kehoe, E. J., \& Marshall, B. S. (1983). Twenty years of classical conditioning research with the rabbit. Progress in Psychobiology \& Physiological Psychology, 10, 197-275.

Harvey, J., Gormezano, I., \& CoOL-Hauser, V. (1983). Effects of scopolamine and methylscopolamine on classical conditioning of the rabbit nictitating membrane response. Journal of Pharmacology \& Experimental Therapeutics, 225, 42-49.

Hasselmo, M. (1995). Neuromodulation and cortical function: Modeling the physiological basis of behavior. Behavioural Brain Research, 67, 1-27.

Hasselmo, M., \& Barkai, E. (1995). Cholinergic modulation of activitydependent synaptic plasticity in the piriform cortex and associative memory function in a network biophysiological simulation. Journal of Neuroscience, 15, 6592-6604.

Hasselmo, M., \& Schnell, E. (1994). Laminar selectivity of the cholinergic suppression of synaptic transmission in rat hippocampal region $\mathrm{CA}$ : Computational modeling and brain slice physiology. Journal of Neuroscience, 14, 3898-3914. 
Hasselmo, M., Schnell, E., \& Barkat, E. (1995). Learning and recall at excitatory recurrent synapses and cholinergic modulation in hippocampal region CA3. Journal of Neuroscience, 15, 5249-5262.

Hinton, G. (1989). Connectionist learning procedures. Artificial Intelligence, 40, 185-234.

HIRSH, R. (1974). The hippocampus and contextual retrieval of information from memory: A theory. Behavioral Biology, 12, 421-444.

HuerTa, P., \& Lisman, J. (1993). Heightened synaptic plasticity of hippocampal CA1 neurons during a cholinergically induced rhythmic state. Nature, 364, 723-725.

IZQUIERDO, I. (1989). Mechanism of action of scopolamine as an amnestic. Trends in Pharmacological Sciences, 10, 175-177.

JACOBS, R. A. (1988). Increased rates of convergence through learning rate adaptation. Neural Networks, 1, 295-307.

JoSEPH, M., Peters, S., \& GRAY, J. (1993). Nicotine blocks latent inhibition in rats: Evidence for a critical role of increased functional activity of dopamine in the mesolimbic system at conditioning rather than pre-exposure. Psychopharmacology, 110, 187-192.

KANEKo, T., \& THOMPSON, R. (1997). Disruption of trace conditioning of the nictitating membrane response in rabbits by central cholinergic blockade. Psychopharmacology, 131, 151-166.

Kaye, H., \& PEARCE, J. (1987). Hippocampal lesions attenuate latent inhibition and the decline of the orienting response in rats. Quarterly Journal of Experimental Psychology, 39B, 107-125.

KESNER, R. (1988). Reevaluation of the contribution of the basal forebrain cholinergic system to memory. Neurobiology of Aging, 9 , 609-616.

Knapp, M., Knopman, D., Solomon, P., Pendlebury, W., Davis, C., \& GRACON, S. (1994). A 30-week randomized controlled trial of highdose tacrine in patients with Alzheimer's disease. Journal of the American Medical Association, 271, 985-991.

KRNJEVIC, K. (1975). Acetylcholine receptors in vertebrate CNS. In L. Iversen, S. Iversen, \& S. Snyder (Eds.), Handbook of pharmacology (Vol. 6, pp. 97-126). New York: Plenum.

KRNJEVIC, K., \& ROPERT, N. (1982). Electrophysiological and pharmacological characteristics of facilitation of hippocampal populations spikes by stimulation of the medial septum. Neuroscience, 7, 2165-2183.

Kronforst-Collins, M., Moriearty, P., RalPh, M., BeCKer, R., SCHMIDT, B., THOMPSON, L., \& DisteRHOFT, J. (1997). Metrifonate treatment enhances acquisition of eyeblink conditioning in aging rabbits. Pharmacology, Biochemistry \& Behavior, 56, 103-110.

Lee, M., Chrobak, J., Sik, A., Wiley, R., \& Buzsáki, G. (1994). Hippocampal theta activity following selective lesion of the septal cholinergic system. Neuroscience, 62, 1033-1047.

LeVY, W. B., Brassel, S. E., \& MoORE, S. D. (1983). Partial quantification of the associative synaptic learning rule of the dentate gyrus. Neuroscience, 8, 799-808.

Lewis, J., LoTurCo, J., \& Solomon, P. (1987). Lesions of the middle cerebral peduncle disrupt acquisition and retention of the rabbit's classically conditioned nictitating membrane response. Behavioral Neuroscience, 101, 151-157.

LOCKHART, M., \& MOORE, J. (1975). Classical differential and operant conditioning in rabbits (Oryctolagus cuniculus) with septal lesions. Journal of Comparative \& Physiological Psychology, 88, 147-154.

LuBow, R. (1973). Latent inhibition. Psychological Bulletin, 79, 398-407.

MACKINTOSH, N. (1973). Stimulus selection: Learning to ignore stimuli that predict no change in reinforcement. In R. Hinde \& J. StevensonHinde (Eds.), Constraints on learning: Limitations and predispositions (pp. 75-96). New York: Academic Press.

MAdison, D., LANCASTER, B., \& NiCOLL, R. (1987). Voltage clamp analysis of cholinergic action in the hippocampus. Journal of Neuroscience, 7, 733-741.

ManNing, F. (1994). Tacrine therapy for the dementia of Alzheimer's disease. American Family Physician, 50, 819-826.

Matzel, L., Schachtman, T., \& Miller, R. (1988). Learned irrelevance exceeds the sum of CS-preexposure and US-preexposure deficits. Journal of Experimental Psychology: Animal Behavior Processes, 14, 311-319.

MEYER, J., ALLEN, D., \& YOKEL, R. (1996). Hippocampal acetylcholine increases during eyeblink conditioning in the rabbit. Physiology \& Behavior, 60, 1199-1203.

Miyamoto, M., Narumi, S., Nagaoka, A., \& Coyle, J. (1989). Effects of continuous infusion of cholinergic drugs on memory impairment in rats with basal forebrain lesions. Journal of Pharmacology \& Experimental Therapy, 248, 825-835.

Moore, J., Goodell, N., \& Solomon, P. (1976). Central cholinergic blockade by scopolamine and habituation, classical conditioning, and latent inhibition of the rabbit's nictitating membrane response. Physiological Psychology, 4, 395-399.

Mosko, S., LynCh, G., \& Cotman, C. (1973). The distribution of septal projections to the hippocampus of the rat. Journal of Comparative Neurology, 152, 163-174.

Myers, C. E., Ermita, B. R., Harris, K., Hasselmo, M., Solomon, P., \& GLUCK, M. A. (1996). A computational model of cholinergic disruption of septohippocampal activity in classical eyeblink conditioning. Neurobiology of Learning \& Memory, 66, 51-66.

Myers, C. E., \& Gluck, M. A. (1994). Context, conditioning, and hippocampal rerepresentation in animal learning. Behavioral Neuroscience, $108,835-847$

Myers, C. E., \& GLuCK, M. A. (1996). Cortico-hippocampal representations in simultaneous odor discrimination: A computational interpretation of Eichenbaum, Mathews, \& Cohen (1989). Behavioral Neuroscience, 110, 685-706.

Myers, C. E., Gluck, M. A.. \& Granger, R. (1995). Dissociation of hippocampal and entorhinal function in associative learning: A computational approach. Psychobiology, 23, 116-138.

Port, R., \& PATterson, M. (1984). Fimbrial lesions and sensory preconditioning. Behavioral Neuroscience, 98, 584-589.

Powell, D., Hernandez, L., \& Buchanan, S. (1985). Intraseptal scopolamine has differential effects on Pavlovian eye blink and heart rate conditioning. Behavioral Neuroscience, 99, 75-87.

Powell, D., Milligan, W., \& Buchanan, S. (1976). Orienting and classical conditioning in the rabbit (Oryctolagus cuniculus): Effects of septal area lesions. Physiology \& Behavior, 17, 955-962.

Powell, D., Milligan, W., \& Mull, P. (1982). Lateral septal lesions enhance conditioned bradycardia in the rabbit. Journal of Comparative \& Physiological Psychology, 96, 742-754.

ProKasY, W. (1972). Developments with the two-stage model applied to human eyelid conditioning. In A. H. Black \& W. F. Prokasy (Eds.), Classical conditioning II: Current research and theory (pp. 119147). New York: Appleton-Century-Crofts.

RESCORLA, R., \& WAGNeR, A. (1972). A theory of Pavlovian conditioning: Variations in the effectiveness of reinforcement and nonreinforcement. In A. H. Black \& W. F. Prokasy (Eds.), Classical conditioning II: Current research and theory (pp. 64-99). New York: Appleton-Century-Crofts.

Rumelhart, D., Hinton, G., \& Williams, R. (1986). Learning internal representations by error propagation. In D. Rumelhart \& J. McClelland (Eds.), Parallel distributed processing: Explorations in the microstructure of cognition (pp. 318-362). Cambridge, MA: MIT Press.

Salvatierra, A., \& Berry, S. (1989). Scopolamine disruption of septo-hippocampal activity and classical conditioning. Behavioral Neuroscience, 103, 715-721.

Scavio, M., Clift, P., \& Wills, J. (1992). Posttraining effects of amphetamine, chlorpromazine, ketamine and scopolamine on the acquisition and extinction of the rabbit's conditioned nictitating membrane response. Behavioral Neuroscience, 106, 900-908.

Schmajuk, N., \& DiCarlo, J. (1992). Stimulus configuration, classical conditioning and hippocampal function. Psychological Review, 99, 268-305.

Schmajuk, N., Lam, Y.-W., \& Christiansen, B. (1994). Latent inhibition of the rat eyeblink response: Effect of hippocampal aspiration lesions. Physiology \& Behavior, 55, 597-601.

Schmaltz, L., \& Theios, J. (1972). Acquisition and extinction of a classically conditioned response in hippocampectomized rabbits (Oryctolagus cuniculus). Journal of Comparative \& Physiological Psychology, 79, 328-333.

Sevush, S., Guterman, A., \& Villalon, A. (1991). Improved verbal 
learning after outpatient oral physostigmine therapy in patients with dementia of the Alzheimer type. Journal of Clinical Psychiatry, 52, 300-303.

Soffie, M., \& LamberTy, Y. (1987). Scopolamine disrupts visual reversal without affecting the first discrimination. Physiology \& Behavior, 40, 263-265.

SoLomon, P. (1977). Role of the hippocampus in blocking and conditioned inhibition of the rabbit's nictitating membrane. Journal of Comparative \& Physiological Psychology, 91, 407-417.

Solomon, P., \& GotTfried, K. (1981). The septohippocampal cholinergic system and classical conditioning of the rabbit's nictitating membrane response. Journal of Comparative \& Physiological Psychology, 95, 322-330.

Solomon, P., Groccia-Ellison, M., Flynn, D., Mirak, J., EdWARDS, K., DUnehew, A., \& Stanton, M. (1993). Disruption of human eyeblink conditioning after central cholinergic blockade with scopolamine. Behavioral Neuroscience, 107, 271-279.

Solomon, P., \& MoORE, J. (1975). Latent inhibition and stimulus generalization of the classically conditioned nictitating membrane response in rabbits (Oryctolagus cuniculus) following dorsal hippocampal ablation. Journal of Comparative \& Physiological Psychology, 89, 1192-1203.

Solomon, P., Solomon, S., van der SchaAF, E., \& Perry, H. (1983), Altered activity in the hippocampus is more detrimental to classical conditioning than removing the structure. Science, 220, 329-331.

SPENCER, D., \& LAL, H. (1983). Effects of anticholinergic drugs on learning and memory. Drug Development Research, 3, 489-502.

StumpF, C., Petsche, H., \& Gogolak, G. (1962). The significance of the rabbit's septum as a relay station between the midbrain and the hippocampus: II. The differential influence of drugs upon both cell firing patterns and the hippocampus theta activity. Electroencephalography \& Clinical Neurophysiology, 14, 212-219.

Thal, L., Fuld, P., Masur, D., \& Sharpless, N. (1983). Oral physostigmine and lecithin improves memory in Alzheimer disease. Annals of Neurology, 13,491-496.

Thompson, R. (1972). Sensory preconditioning. In R. Thompson \& J. Voss (Eds.), Topics in learning and performance (pp. 105-129). New York: Academic Press.

Thompson, R. (1986). The neurobiology of learning and memory. Science, 233, 941-947.

Thompson, R., Berger, R., Berry, S., Hoehler, F., Kettner, R., \& WEISZ, D. (1980). Hippocampal substrate of classical conditioning. Physiological Psychology, 8, 262-279.

TOth, K., Borhegyi, Z., \& Freund, T. (1993). Postsynaptic targets of GABAergic hippocampal neurons in the medial septum-diagonal band of Broca complex. Journal of Neuroscience, 13, 3712-3724.

VANDERWOLF, C., \& LeUnG, L.-W. (1983). Hippocampal rhythmical slow activity: A brief history and the effects of entorhinal lesions and phencyclidine. In W. Seifert (Ed.), Neurobiology of the hippocampus (pp. 275-302). London: Academic Press.

WaGSTAFF, A., \& MCTAVish, D. (1994). Tacrine. A review of its pharmacodynamic and pharmacokinetic properties, and therapeutic efficacy in Alzheimer's disease. Drugs \& Aging, 4, 510-540.

WALlenstein, G., \& Hasselmo, M. (1997). GABAergic modulation of hippocampal population activity: Sequence learning, place field development, and the phase precession effect. Journal of Neurophysiology, 78, 393-408.

WeISKRANTZ, L., \& Warrington, E. (1979). Conditioning in amnesic patients. Neuropsychologia, 17, 187-194.

Whitehouse, P., Price, D., Struble, R., Clark, A., Coyle, J., \& DeLONG, M. (1982). Alzheimer's disease and senile dementia: Loss of neurons in the basal forebrain. Science, 215, 1237-1239.

Widrow, B., \& HoFF, M. (1960). Adaptive switching circuits. Institute of Radio Engineers, Western Electronic Show \& Convention Record, 4, 96-104.

WoODRUFF-PAK, D. (1993). Eyeblink classical conditioning in HM: Delay and trace paradigms. Behavioral Neuroscience, 107, 911-925.

WOOdRUFF-PAK, D., LI, Y., \& KEM, W. (1994). A nicotinic agonist (GTS21), eyeblink classical conditioning, and nicotinic receptor binding in rabbit brain. Brain Research, 645, 309-317.
ZACKheim, J., MYers, C., \& GluCK, M. (1997). A recurrent model of the hippocampal-cerebellar substrates of response timing and learned temporal stimulus relationships in sensory motor conditioning. Cognitive Neuroscience Abstracts, 67, 46.

Zola-Morgan, S., SQuire, L., \& Amaral, D. (1989). Lesions of the hippocampal formation but not lesions of the fornix or the mammillary nuclei produce long-lasting memory impairment in monkeys. Journal of Neuroscience, 9, 898-913.

\section{NOTE}

1. Modeling data reported have been averaged over 20 simulation runs; each randomly initialized and then given identical training parameters. Mean, variance, and other statistical computations are calculated from the results of these runs. Full details of the model are given in the appendix.

\section{APPENDIX Simulation Details}

The corticohippocampal model used in these studies is essentially the same as that used in previous implementations (Gluck \& Myers, 1993; Myers et al., 1996; Myers \& Gluck, 1994). The model parameters were optimized to produce a system in which both intact and lesioned models learn a simple conditioned acquisition quickly and at approximately the same speed. The model was not optimized for any other tasks.

\section{The Intact Model}

The intact model consists of a hippocampal region network and a network representing cerebral and cerebellar cortices, as shown in Figure 1A (Gluck \& Myers, 1993). The hippocampal region network is an autoencoder (Hinton, 1989) with 15 input nodes, 8 hidden or internal layer nodes, and 16 output nodes. The cerebellar network is a feedforward network with 15 input nodes, 40 hidden or internal layer nodes, and 1 output node. There is full connectivity between adjacent layers in each network; at the start of a simulation run, all weights and biases are initialized from the random distribution $U(-0.3,+0.3)$.

Initialization and training schedule. On each trial, the model is presented with a 15 -element input string, representing the presence or absence of up to five CSs and 10 background or contextual elements. This string becomes the activation for the input layer nodes in both the hippocampal region and the cerebellar networks. The networks process this information (described below), and the activation of the cerebellar network output node represents the strength of the $C R$ in response to those inputs. This is then compared against a binary signal representing the presence or absence of the US on the current trial.

At the start of each simulation, the network weights are initialized. Next, the model output is initialized by 500 trials of training on an all-0 input string with no US present; this ensures that all simulations (despite variable initial conditions) give consistent no-CR responses to initial CS presentations. The contextual elements are then each randomly initialized to 0 or 1 , and they are thereafter held constant for the duration of a simulation run. At this point, training begins. The model is given trials with appropriate CS and US presentations; these trials are intermixed with context-only (no-CS or US) trials in a 1:20 ratio. Over many trials, the model learns to generate a CR in response to CSs that have been repeatedly paired with the US (see, e.g., Figure 6B). Because of variable initial conditions, two simulation runs may differ slightly in performance measures such as speed of learning or strength of the CR. Thus, all results reported in this paper are averaged over 20 simulation runs. Sta- 
tistical tests have been used to ensure that performance is robust and reliable across simulations given similar training: thus, for example, the intact simulations in Figure $6 \mathrm{~B}$ learn the CR reliably faster than do the scopolamine simulations.

The hippocampal region network. Nodes in the hippocampal region network are activated as a function of node activity in the previous layer. Thus, for all nodes $j$, activation $y_{j}$ is computed as

$$
\begin{aligned}
y_{j} & =f\left(\sum_{i} w_{i j} y_{i}+\theta_{j}\right) \\
f(x) & =\frac{1}{\left(1+e^{-x}\right)},
\end{aligned}
$$

where $w_{i j}$ is the weight from node $i$ to node $j$ and $\theta_{j}$ is the bias of node $j$. The output nodes are trained to reproduce the input vector plus a prediction of US arrival on the current trial. To accomplish this, weights in the network are trained via the error backpropagation algorithm (Rumelhart et al., 1986). Following each trial, error signals for the output nodes $j$ are calculated by

$$
\delta_{j}=\left(I_{j}-y_{j}\right) y_{j}\left(1-y_{j}\right)
$$

where $I_{1}$ through $I_{15}$ are the 15 external inputs, and $I_{16}$ is 1.0 if the US is present on the current trial and 0.0 otherwise. Error signals for internal nodes $j$ are calculated as

$$
\delta_{j}=y_{j}\left(1-y_{j}\right)\left(\sum_{k} w_{j k} \delta_{k}\right) .
$$

Weights are then incremented with $\Delta w_{i j}=\alpha\left(\Delta w_{i j}{ }^{\prime}\right)+\beta \delta_{j} y_{i}$, where $\Delta w_{i j}{ }^{\prime}$ was the increment to $w_{i j}$ on the previous trial. In the intact model, the hippocampal region network's learning rate is $\beta=0.05$ on trials when the US is present; this is reduced to $\beta=0.005$ on trials when the US is absent. Momentum is set at $\alpha=0.9$. Biases are trained as if they were weights from constantly active inputs.

Cerebellar network. Node outputs $y_{j}$ are computed as in the hippocampal region network. After each trial, the error signal for the output node $\delta_{j}$ is US-CR; the error signals for the internal layer nodes are computed as $d_{j}=y_{k}-y_{j}$, where $y_{k}$ is the output of the $(j \bmod 8)$ th internal layer node in the hippocampal region network. Thus, each hippocampal network hidden layer node provides the training signal for 10 cerebellar network hidden nodes. Weights for all cerebellar network nodes are then incremented according to the LMS algorithm (Widrow \& Hoff, 1960): $\Delta w_{i j}=\eta \delta_{j} y_{i}$. The learning rate is $\eta=0.5$ for the output layer node and $\eta=0.1$ for internal layer nodes; again, learning rates are decreased tenfold on trials when the US is absent.

The activity of the output node is rescaled to give a baseline activity of 0.0 and reported as the probability of a CR; this value is averaged across simulations to give the percent $C R$ measure shown in the graphs.

\section{The Lesioned Model}

The lesioned model is as shown in Figure 1B. The hippocampal region network is disabled, and the error signals to the cerebellar network internal layer nodes are fixed at $\delta_{j}=0$. Thus, no further modifications are made to the lower layer of weights in the cerebellar network. The upper layer of weights are trained as in the intact model, allowing the lesioned model to learn to map from existing representations to new behavioral responses.

\section{The Scopolamine Model}

The scopolamine model is the same as the intact model, except for a lowered learning rate in the hippocampal region network: $\beta=0.005$ on US-present trials and $\beta=0.0005$ on USabsent trials.

\section{The Physostigmine Model}

The physostigmine model shown in Figure $7 \mathrm{C}$ has a very high hippocampal region network learning rate ( $\beta=1.0$ on USpresent trials and $\beta=0.1$ on US-absent trials) and represents conditioning with very high doses of cholinergic agonists such as physostigmine.

(Manuscript received December 10, 1996; revision accepted for publication December 22, 1997.) 